Aided Design

Elsevier Editorial System(tm) for Computer-

Manuscript Draft

Manuscript Number: CAD-D-13-00070R1

Title: Footwear Bio-modelling: An industrial approach

Article Type: Full Length Article

Keywords: deformation techniques; biodeformable modelling; customised footwear; footwear production

Corresponding Author: Mr. Antonio Jimeno-Morenilla, Ph.D.

Corresponding Author's Institution: University of Alicante

First Author: Miguel Davia, Ph.D.

Order of Authors: Miguel Davia, Ph.D.; Antonio Jimeno-Morenilla, Ph.D.; Faustino Salas

Abstract: There is a growing need within the footwear sector to customise the design of the last from which a specific footwear style is to be produced. This customisation is necessary for user comfort and health reasons, as the user needs to wear a suitable shoe. For this purpose, a relationship must be established between the user foot and the last with which the style will be made; up until now, no model has existed that integrates both elements. On the one hand, traditional customised footwear manufacturing techniques are based on purely artisanal procedures which make the process arduous and complex; on the other hand, geometric models proposed by different authors present the impossibility of implementing them in an industrial environment with limited resources for the acquisition of morphometric and structural data for the foot, apart from the fact that they do not prove to be sufficiently accurate given the non similarity of the foot and last. In this paper, two interrelated geometric models are defined, the first, a bio-deformable foot model and the second, a deformable last model. The experiments completed show the goodness of the model, with it obtaining satisfactory results in terms of comfort, efficiency and precision, which make it viable for use in the sector. 


\title{
Footwear Bio-modelling: An industrial approach
}

\begin{abstract}
There is a growing need within the footwear sector to customise the design of the last from which a specific footwear style is to be produced. This customisation is necessary for user comfort and health reasons, as the user needs to wear a suitable shoe. For this purpose, a relationship must be established between the user foot and the last with which the style will be made; up until now, no model has existed that integrates both elements. On the one hand, traditional customised footwear manufacturing techniques are based on purely artisanal procedures which make the process arduous and complex; on the other hand, geometric models proposed by different authors present the impossibility of implementing them in an industrial environment with limited resources for the acquisition of morphometric and structural data for the foot, apart from the fact that they do not prove to be sufficiently accurate given the non similarity of the foot and last. In this paper, two interrelated geometric models are defined, the first, a bio-deformable foot model and the second, a deformable last model. The experiments completed show the goodness of the model, with it obtaining satisfactory results in terms of comfort, efficiency and precision, which make it viable for use in the sector.
\end{abstract}




\section{Reviewer \#1}

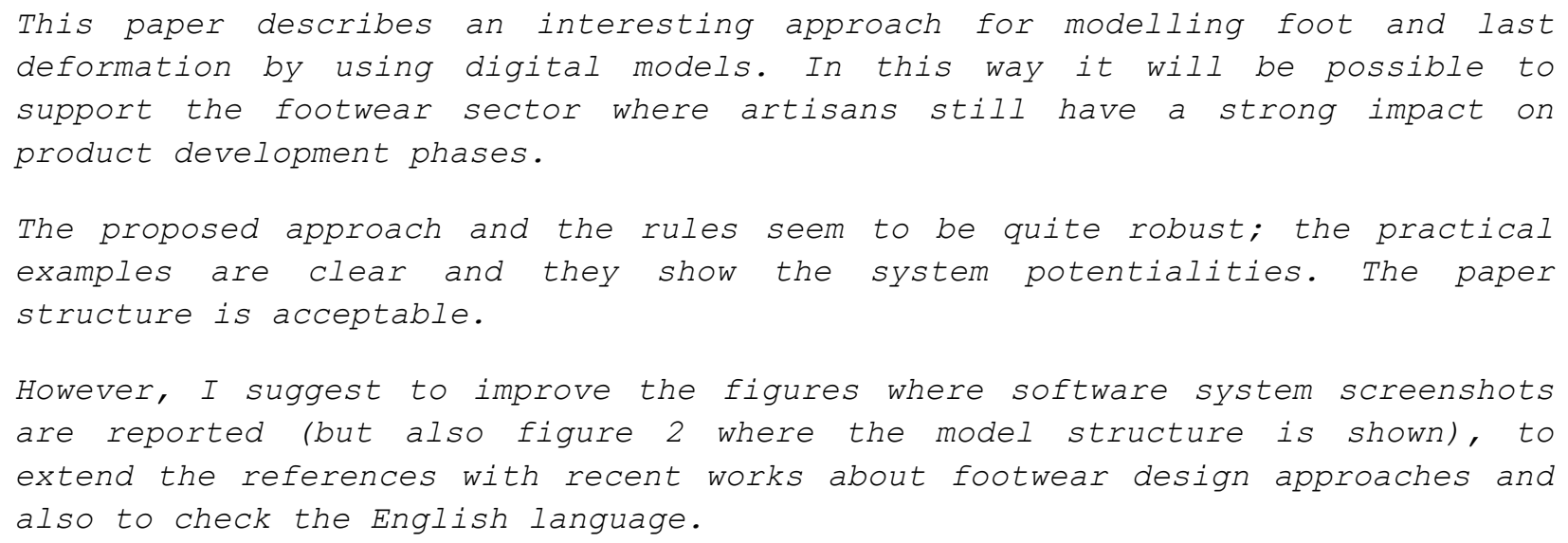

Finally I think this paper is acceptable with minor revisions

- The quality of the figures is reduced in the online process for file conversion into PDF. For this reason, the figures cited by the reviewer have been also separately included in this version. This way, the reviewer can get high-quality figures.

- The English language has been checked.

- According to the reviewer's suggestions, references [10], [11] and [12]:have been added

1. [10] Advanced computer aided technologies for design automation in footwear industry, International Journal on Interactive Design and Manufacturing (IJIDeM), August 2011, Volume 5, Issue 3, pp 137-149, R. Raffaeli, M. Germani

2. [11] Shoes customization design tools for the diabetic foot, M Davia, M Germani, M Mandolini, M Mengoni, E Montiel, R Raffaeli, Computer-aided Design \& Applications 8 (5), 693-711

3. [12] CAD tools for designing shoe lasts for people with diabetes, Original Research Article Computer-Aided Design, In Press, Corrected Proof, Available online 11 February 2013. José Antonio Bernabéu, Michele Germani, Marco Mandolini, Maura Mengoni, Chris Nester, Steve Preece, Roberto Raffaeli

\section{Reviewer \#2}

The paper is well written and has very good points.

1. The main concern is the accuracy of deformations especially when considering foot shape from flat heel to high heel and with toe spring.

of course we can use many different methods to modify the surface but how good is it to reality.

- There are many different possibilities to adapt the foot surface to the last pitch (heel height). Cage-based methods (exoskeletons) perform better than others when it comes to the deformation of the surface, in that the cage can be better controlled and adapted to the foot morphology, allowing bending areas to be more accurately defined according to the characteristic curves of the foot. The paper rules out Free Form Deformation (FFD) based methods and skeleton based methods, since the deformer do not allow deformation areas to be 
properly defined due to their cubic structure, and also due to the fact that their internal vertexes prevent the adequate control of the foot geometry. Furthermore, the skeleton (endoskeleton) based methods require the prior obtaining of the bone structure of the foot in order to start the setup process of the skeleton, a process that can be complex in an industrial setting as it requires the use of certain devices that enable the detection of the bone structure of the foot.

2. Only one test has been done. Based on my experience, I know that one test does not mean much when considering design for the general population. One test is heavily biased.

- The authors have carried out many tests, but they decided to include just one example so as to be able to provide detailed explanations and not to extend the paper too much. This example was chosen because it illustrated a difficult adaptation of the shoemaking last design, which is always solved manually by expert last makers with the corresponding high economic and time cost.

- Nevertheless, and with a view to demonstrating the versatility of the model, we have included figures 32 and 33 (on page 17) along with a series of models and the main modifications made. For length reasons, each of the modification processes carried out have not been explained in detail.

\section{Reviewer \#4}

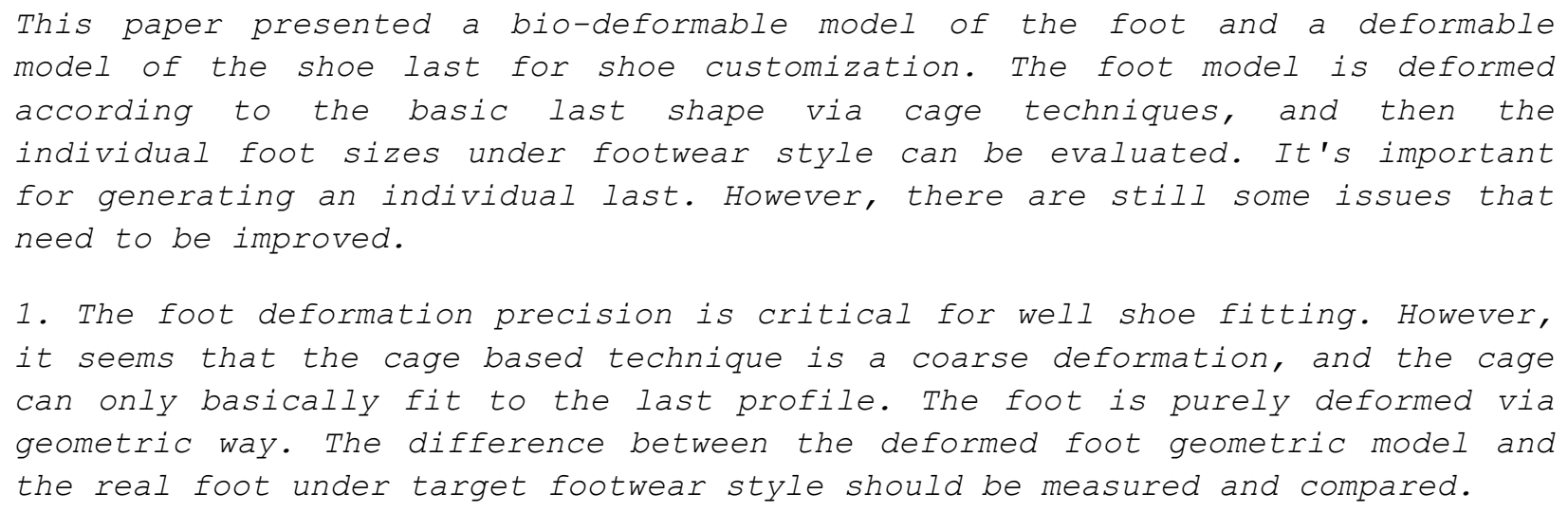

- This paper presents a merely industrial approach. The introduction accounts for the dismissal of musculoskeletal models (undoubtedly, more accurate) due to the impossibility of working with them for their high computational and the high economic cost of the necessary devices.

- The deformation of the foot does not take soft areas into account, and the joints are controlled via the cage. Obviously, the digitally deformed foot is not exactly the same as the real flexed foot would be. However, this deformation enables:

$\circ$ a sole foot geometry acquisition, instead of multiple geometry acquisitions for each heel height;

- speed of the automatic deformation, that can be completed using conventional PCs;

$\circ$ an acceptable measurement between the shoe last and the deformed foot to be ensured.

- In order to prove that this deformation is sufficient according to industrial manufacturing criteria, table 2 has been added (on page 9), which shows the real differences and the error 
between the digital foot deformed geometrically and the foot digitised at typical heel heights of 40,60 and $80 \mathrm{~mm}$. As you can see, the differences in the measurements that are taken as an international reference in industrial design are minimal. Such measurements include critical points with more variability, and will be used as specific measurements to build the shoe last.

2. The fitting test is somehow simple.

(1) How many people are tested? How about the proportion of young or old, man or woman, etc?

- The authors have carried out many tests, but they decided to include just one example so as to be able to provide detailed explanations and not to extend the paper too much. This example was chosen because it illustrated a difficult adaptation of the shoemaking last design, which is always solved manually by expert last makers.

- There is a prototype "custom footwear store" in INESCOP premises where many feet are scanned every day for the subsequent production of customised footwear. This is obviously a prototype that needs some fine-tuning before becoming an industrial process, but the results are highly satisfactory to the users.

- The type of people that have used this prototype is quite varied: men, women, children and elderly. It has also been tested with people suffering from foot conditions, such as diabetic feet (see the bibliographic references on this issue).

(2) How about the fit evaluation of the shoe that is designed in the traditional way?

- The tests were carried out in the traditional way using footwear samples. The footwear maker tries the footwear sample on the tester and the same parameters as those listed in the tables in this paper are assessed.

(3) The paper only provides one footwear style for fitting test. It'll be better if the authors can provide more design results and fitting tests, which can show the effects of the proposed method more clearly.

- $\quad$ With a view to demonstrating the versatility of the model, we have included figures $\mathbf{3 2}$ and 33 (page 17) along with a series of models and the main modifications made. For length reasons, each of the modification processes carried out have not been explained in detail.

\section{Reviewer \#5}

The modelling involved another algorithm. The authors claim quite a lot in terms of efficiency, effectiveness and comfort. In my opinion, they have very little knowledge about comfort. Even the prototype produced is a very low heel shoe. A high heel shoe would be at least $50 \mathrm{~mm}$ high.

The topic of comfort has been investigated by many and their claim that it is impossible to know the interaction between foot and shoe is not true. 
Introduction: Page 1: all texts related to FA are meaningless. Focus on the issue at hand. The authors need to look at the ergonomics literature and especially focus on labs in Hong Kong (Goonetilleke), Germany (Krauss) and spain (Alemany). An useful website that the authors should look into is: http://www.ielm.ust.hk/dfaculty/ravi/papers_all.html.

There are many related papers and I give below a few that are accessible.

Goonetilleke, R.S. (Ed.) (2012) Science of Footwear. CRC Press 1-726

Witana, C. P., Goonetilleke, R. S., Au, E. Y. L., Xiong, S. and Lu, X. (2009)

Footbed shapes for enhanced footwear comfort. Ergonomics, Vol. 52(5), May $2009,617-628$.

Witana, C.P., Xiong, S., Zhao, J. and Goonetilleke, R. S. (2006) Foot measurements from three-dimensional scans: A comparison and evaluation of different methods. International Journal of Industrial Ergonomics September, Vol. 36(9), pp. 789-807.

The book on science of footwear is very relevant, especially for the measurements. The key to comfort is the footbed shape and I urge the authors to look at the related patents of Goonetilleke and his papers before any algorithm can be developed. It is easy to develop an algorithm but not that easy to develop an algorithm based on human sensation. In my opinion, what is in the manuscript is another algorithm and unless the ergonomics is incorporated, the algorithm is not very useful.

We thank the reviewer for his comments about comfort. Comfort is a broad concept that embraces a wide spectrum of knowledge, not only ergonomic, but also sensory and even emotional knowledge. As stated by the reviewer, this is quite a complex concept and many have investigated this topic addressing footwear comfort from different points of view, either by defining comfort aspects with regard to the foot-bed, the materials used, or the outsole, insole, etc. This wide range of factors makes the industrial footwear manufacturing process complex, in the same way as the achievement of the best possible fit.

The authors agree with the reviewer that, even though there are many factors involved in comfort, it is still possible to achieve the ideal interaction between foot and shoe. It is also true that there is still a long way to go. A proof of this is that there is no comprehensive solution to the footwear design issue in the literature wisely provided by the reviewer or in other unreferenced literature, but instead there are only partial solutions that affect the footbed, the last pitch, etc.

However, this paper is not intended to find the ideal relationship between foot and shoe. From the very beginning, in the title, the authors stressed the "industrial approach". We are all researchers closely linked to the footwear industry through the Spanish Footwear Technological Institute (INESCOP). Not for nothing, INESCOP is the leading institution worldwide for research in footwear technology. Its CAD/CAM products are leading solutions for footwear design and manufacture. Therefore, we have extensive knowledge of the industrial sector addressed by this paper. The term "comfort" as used in this paper is related to the first stage of the footwear design process, which is the production of the shoe last and not the production of other footwear components, such as the sole, the insole, or the raw materials. Obviously, a shoe with a perfect geometric adaptation to the user's foot can be extremely uncomfortable if the adequate materials are not used, but this is not the issue addressed in this paper. 
As the reviewer may know, footwear is currently designed based on average population measurements without taking the individual user into account. It can be stated that the footwear available on the market, arranged by sizes, either fits everybody or does not fit anyone. These shoes are industrially produced using an object that resembles the shape of the foot: the shoemaking last.

The current problems of the footwear industry with regard to the design of shoe lasts are as follows: given a new market trend for the design of new footwear styles, the highest possible precision is required when it comes to designing suitable lasts for such styles. The process performed by last makers is based on the selection of the body of a base last, which is deemed to have the adequate sole according to the last maker's expertise, but then the pitch, the toe and other aspects related to its upper surface are changed according to the intended shoe style to be produced. Therefore, in order to know if the changes to be made are correct or not, it is necessary to compare the measurements with those of the model foot that matches the last pitch or heel height. Such measurements are those described in the paper. The use of these measurements and the modification of the last are completed by last makers according to their expert criteria, which is not addressed in this paper.

The proposed model is not an algorithm. Instead, it is a design system that includes the various components involved in the industrial footwear manufacturing process, but it has a brand new feature: the morphology of the user's foot is taken into account. The components involved in the model are: digitised feet, CAD-designed lasts, an international measurement system, a set of geometric deformation tools for feet and lasts, and finally, an expert last maker who makes the decisions. Therefore, it cannot be stated that this set of elements represent "just another algorithm"; it is a new way of designing footwear aimed to produce customised footwear on an industrial level.

INESCOP, representing AENOR (Spanish Association for Standardisation and Certification), is participating in the preparation of an international standard that will enable the worldwide harmonisation of sizing systems within the technical committee ISO/TC 137 - Footwear sizing designations and marking systems. This committee is defining the characteristic (most relevant) measurements of the foot and the last. It should be noted that there is great variability in the ways in which each member country defines these measurements, whether it be in Spain, France, Italy, Germany, UK, Czech Republic, China, South Africa, etc... The footwear manufacturing tradition in each country entails different ways to measure lasts and feet; therefore, the reviewer's attention is drawn to the fact that it is really difficult to define a standard that joins all considerations and necessary mechanisms to correctly define the suitable measurements that characterise a foot or a last.

In an industrial setting, last makers rely on their extensive experience and the successful design of lasts that have resulted in big sales and wide acceptance on the market. The studies wisely suggested by the reviewer precisely rationalise the design process, laying the foundations in terms of comfort, but they can hardly be applied in real manufacture without the suitable CAD support that enables the effective comparison between foot and last in order to modify the original last based on the analysis of the results. Moreover, the comfort terms mentioned in those studies may be applied with difficulty in highheeled women's shoes with a sharp toe shape that excessively compresses the toes.

The model created by the authors has been implemented in the most widely used design software for lasts, which is currently being used by the main last makers worldwide. This model makes it possible to include any of the characteristics mentioned in the suggested studies, since the model is completely parameterised and allows further fine-tuning, which will be undertaken in the future. By way of 
example, it could be noted that it is possible to modify the shape of the last sole according to the comfort criteria established in the above-mentioned studies.

Finally, the authors would like to state that further basic research is needed to elucidate the foot-shoe relationship, according to purely ergonomic criteria, so as to be able to achieve automatic or semiautomatic design models that allow the production of the most comfortable possible footwear. However, it is also really important that the industry takes more or less ambitious, but also realistic and usable steps that enable the manufacture of more and more comfortable and quality footwear conceived for a particular user and not for a population average. This has been the rationale behind our research. 
Footwear Bio-modelling: An industrial approach

Highlights

- We present two interrelated models for shoe customization.

- We present a bio-deformable model of the foot.

- We present a deformable model of the shoe last.

- $\quad$ Both models are used to successfully make customized shoes. 


\title{
Footwear Bio-modelling: An industrial approach
}

\author{
Miguel Davia ${ }^{1,2}$ mdavia@dtic.ua.es \\ Antonio Jimeno-Morenilla ${ }^{1}$ jimeno@dtic.ua.es \\ Faustino Salas ${ }^{2}$ fsalas@inescop.es \\ ${ }^{1}$ University of Alicante, Department of Computer Technology, Ctra. San Vicente del Raspeig S/N, Alicante (Spain) \\ ${ }^{2}$ Footwear Technological Institute (INESCOP), Polígono Industrial. Campo Alto, Elda, Alicante (Spain)
}

\begin{abstract}
There is a growing need within the footwear sector to customise the design of the last from which a specific footwear style is to be produced. This customisation is necessary for user comfort and health reasons, as the user needs to wear a suitable shoe. For this purpose, a relationship must be established between the user foot and the last with which the style will be made; up until now, no model has existed that integrates both elements. On the one hand, traditional customised footwear manufacturing techniques are based on purely artisanal procedures which make the process arduous and complex; on the other hand, geometric models proposed by different authors present the impossibility of implementing them in an industrial environment with limited resources for the acquisition of morphometric and structural data for the foot, apart from the fact that they do not prove to be sufficiently accurate given the non similarity of the foot and last. In this paper, two interrelated geometric models are defined, the first, a bio-deformable foot model and the second, a deformable last model. The experiments completed show the goodness of the model, with it obtaining satisfactory results in terms of comfort, efficiency and precision, which make it viable for use in the sector.
\end{abstract}

Keywords. Deformation techniques, Customised footwear, Footwear production.

\section{Introduction}

Footwear comfort is one of the most important aspects to be taken into account by footwear production companies $[1,2]$. Comfort is perceived by users as directly related to the successful purchase of a product and also the medium and long term health effects of the product. The perceived comfort analysis of footwear use has been addressed through different approaches [3,4,5], many of these focus on the fit analysis of the footwear style, such as in [6], in which the problem of women's footwear fitting was addressed, where the aesthetic considerations of the model have special effects on the fit evaluation of the shoe. Footwear users increasingly demand products with improved performance $[7,8]$ with variety of designs and renewal of collections on the market [9]. This market revolution acts as stimulation for the footwear industry that is being modernised through the introduction of CAD technology in its processes, which allows the sector to save costs and time in the development of a product. The suitability of footwear for a specific foot with any kind of inherent problem, whether this be major or minor, is the main problem for made-to-measure footwear manufacturers, as there is currently no parametric model that is capable of self configuration of design according to reference feet.

Recent studies define CAD tools used to create custom shoes for general [10] and specific medical issues, such as for diabetic patients, who suffer from the disease called "diabetic foot" $[11,12]$. There are a wide range of studies on musculoskeletal models $[13,14,15,16,17,18,19,20,21]$. There are also a number of scientific contributions being made to the study of computer graphics, some exclusively centred around human muscular anatomy and not on dynamic simulation, such as $[22,23,24,25,26]$. These authors have developed volumetric muscular models in order to simulate muscle behaviour through active and passive components. In [27] an elastic linear model is used for muscle simulation and deformation using finite element methods. In $[16,28]$ a muscular model was created to animate virtual models of the human body (character animation). Musculoskeletal models have also been widely used for facial animation [29,30,31]. In [32] a structural model of the human anatomy is proposed based on deformation through the articulation points of human body virtual models. In [7] a neuro-muscular control model is used for the simulation of the human knee.

The first studies in which the characterisation and simulation of the lower limbs and the interconnection of the skeletal and muscular behaviours were attempted, were those by [33], who developed a musculoskeletal model of the human lower extremity to allow computer simulation. This model incorporates the salient features of muscle and tendon, specifies the musculoskeletal geometry and musculotendon parameters of eighteen musculotendon actuators, and defines the active isometric moment about the knee and ankle joints. In [34], the effect of the human foot's tendons on the deformation of the foot surface was analysed. This stresses the importance that modelling has, not only of muscles and bones, but tendons as well as there are also certain forces that have effects on the system's modelling and simulation, affecting the final foot surface deformation. Later research by these authors [35] widened ligament-tendon modelling using forces related to curves and mass-springs that deform the foot surface, simulating the tendon's position. 
There are contributions related to the simulation of plantar pressures obtained through the application of force on a foot [36] in which, through finite element techniques, a foot is modelled according to the pressures measured on the foot plantar area, taking the influence of the foot's bones and the application of a certain force into account.

In the customised footwear manufacturing process, there is a challenge that makes these models difficult to apply in the industry. In the previously mentioned models, the anatomical information of the bone structure of the limb to be deformed is needed, including the muscular-tendon representation of the foot, and this requires high cost equipment to be available, such as a CT scanner, as well as the software for the reconstruction and later linking of the geometry represented by the skeleton and the foot surface in order to carry out the deformation. It is therefore necessary to include the following constraints in the definition of the model: Efficiency, in the acquisition of the foot geometry with a short timeframe and at a low cost; Effectiveness, for the application of deformation techniques that make the deformation and positioning of the foot possible with the available information and subject to the previous constraint.

The models proposed in this paper meet the precision, flexibility and efficiency requirements needed by the footwear industry. The model presents two clearly differentiated aspects to meet the requirements established by the footwear customisation process: firstly, the deformation of the foot for its adaptation to the sole profile curve (pitch) of the last with which the comparison is made; and secondly, the deformation of the last according to biomechanical criteria related to the reference foot.

This paper is structured as follows: Section 2 analyses the problem of suitability of the last for the foot, critically reviewing various works on the topic; Section 3 defines the geometric models: on the one hand, the bio-deformable foot model, which is characterised in terms of the morphological information of the model and is deformed according to such information, and on the other hand, the deformable last model, characterising the last and defining a group of tools that allow the controlled modification of the last. The deformation and modification operations carried out for the foot and last respectively are then translated to the production of customised footwear. Section 4 presents a real case carried out in real production environments, which proves the goodness of the models.

\section{The problem of suitability of the last for the foot}

The last, the main element in footwear production, constitutes the base for the footwear lasting process: The way in which the user perceives the necessary level of comfort to wear a pair of shoes and carry out his/her daily activities depends greatly on the last. The last is a geometric body with similar (but not equal) dimensions to those of the foot, and certain factors have to be taken into account which, if correctly defined, allow the design of the last.
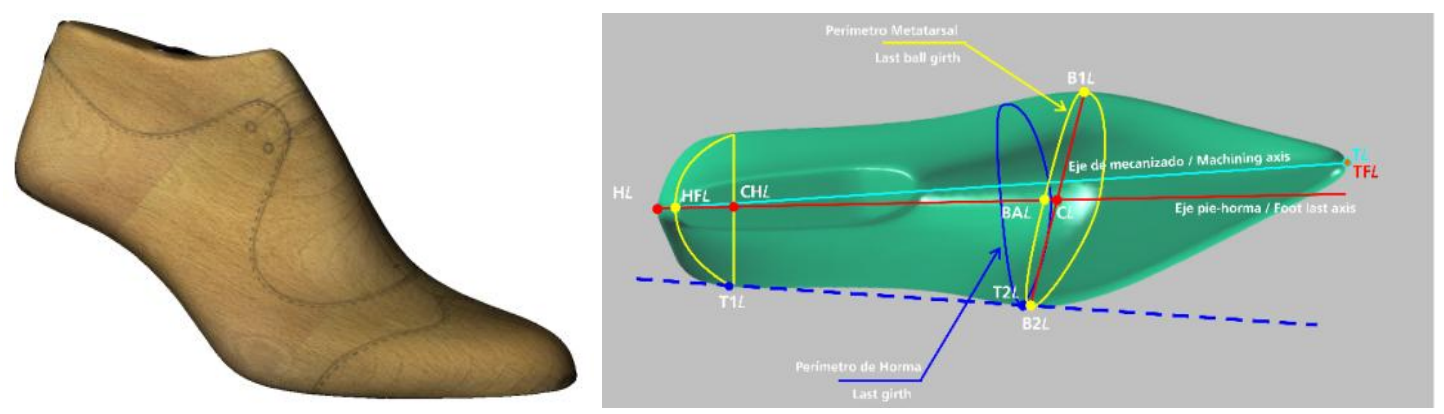

Fig. 1. The last and its measurements

There is a set of measurements that are obtained from characteristic points or landmarks on the last which allow the approximate definition of the last (see Fig 1). These measurements characterise the last quantitatively, providing useful information for comparison with feet. However, one cannot be certain that a determined set of measurements provides the best possible last, in terms of fitting, since there are certain external factors, such as the type of footwear being made, the use of the footwear and the type of reference foot, which constitute a source of uncertainty that requires a different kind of analysis. There are a number of requirements related to footwear fit, which means that the shoe does not have to be totally fitted for the foot [6,37]. The footwear lasts are narrower in certain areas than feet [38], which does not cause pain or discomfort in the foot due to the foot's ability to vary its physiological structure and adapt itself to the inside of the shoe. Different authors have attempted different approaches in the last few years in order to parameterise the design of a given last with regard to the surface of the foot. In [39] the modification of the last through space deformation techniques based on Free Form Deformation (FFD) was presented, in order to compare certain characteristic measurements of the reference foot with the last. This deformation approach was insufficient, as FFD cannot correctly define the in-shoe deformation of a foot. Also, the last deformation carried out does not guarantee correct fitting as a whole, as the anatomical characteristics of the original last can be lost (see Fig. 2). 


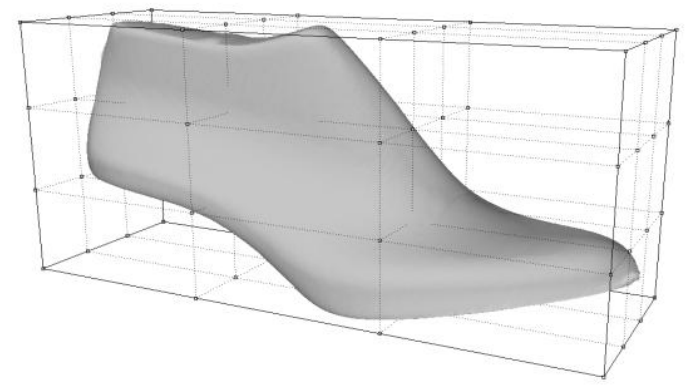

Fig. 2. FFD deformation of the last. Assignment of a control mesh

In other works, systems were inferred to guarantee better fitting between the foot and last, such as the work by [40] in which certain measurements were compared through comparisons between a reference foot and a database with feet linked to lasts. In this approach, the digitised reference foot is positioned with regards to the other foot and compared through the calculation of the error function of the weighted distances. This requires a pre-adjustment between the lasts and reference feet to be compared, although this cannot guarantee that the fitting obtained will be correct. In the work by [41] the determination of certain key points on the foot sole which need to be taken into account to carry out the fitting, is considered. This method establishes a basic definition for the acquisition of the footprint of the sole from the detection of certain characteristic points. The fitting process is restricted to the sole of the foot and does not consider the volume as a whole, thus losing valuable information in the process, which is needed to guarantee correct fitting. In [42] general volumetric deformation methods are included for the adaptation through distance maps between objects. In this work, the last is deformed according to the distance to the reference foot, deformation which intends to approximate the last to the foot without substantial modifications of the basic structure of the shoe last. This method is not efficient for customised footwear manufacture, in that the resulting lasts can end up being aesthetically incorrect and may not guarantee correct fitting, as a last does not completely correspond to a certain foot's geometry. In [43] a basic last is obtained from the foot surface through the transformation of the surface using deformation techniques based on the Laplacian operator [44]. This method poses the problem of the planar acquisition of the foot geometry and compares it with that of a last the sole of which does not lie completely flat on the ground; hence information relevant to the fitting of a shoe is omitted. Similarly, in [45] a method is proposed for the analysis of the suitability of a last to a certain foot, through the comparison of a reduced number of sections obtained from both the foot standing flat and the last. However, it is noticed that in this work, the tests were carried out with a low-heeled last and the aesthetic shape of its toe is characterised by smooth shapes. With this type of approach, through the analysis of a flat foot and a last with low pitch, a wide range of lasts, which are typically highly demanded in high-end footwear, is neglected.

In contrast to the previously mentioned works, in which the models are basically static, this paper proposes a different approach for the resolution of the problem with high-level footwear customisation as the fit is achieved dynamically.

The foot is normally digitised in a resting position, supported by the ground plane, with no heel height. However, the lasts which are later used to last the footwear do have a heel height, thus making the comparison difficult. Matching a foot to a last if the foot is not adapted to the plantar curve defined by the last is a complex task. It is necessary for the foot to take the sole shape of the last and be placed at the corresponding heel height in order to make a comparison. Making this modification in the foot's original geometry requires a biomechanical study of the foot, defining how certain areas of the foot deform and articulate. The achievement of the best possible fit between foot and last is the goal, as, in the fitting of a shoe, the suitability of the last to the reference foot is a decisive factor when a user decides to buy a pair of shoes, and is therefore fundamental in the perception of comfort [46,47,48]. To date, it has been impossible to know how the dimensions and footwear shape and its interaction with the foot affect subjective comfort perceived by the user $[2,49]$.

\section{Geometric bio-deformable model for footwear design}

Below, each of the elements that form part of the model is characterised. In an industrial environment, the starting point for the customised footwear production process is the creation of an industrial element $E_{\text {base }}$, called the last, which is used as an indispensable and fundamental element for the production of an $E_{\text {final }}$ industrial element, called the shoe, that must meet a certain set of quality criteria $C_{\text {bio-base }}$ which guarantee the proper suitability of the product to the final user. In order to pass these quality controls, $E_{\text {final }}$ must be manufactured using $E_{\text {base }}$, which in itself is subject to a set of restrictions $R_{\text {base }}$ given by a biological element, $E_{b i o}$, called the foot, which belongs to a biological system, $S_{\text {bio }}$, where a set of restrictions, $R_{b i o}$, can be found which biomechanically characterise its structure and functionality. 
Table 1

Model elements

\begin{tabular}{ll}
\hline $\boldsymbol{S}_{\text {bio }}$ & Biological system which includes the biological reference element \\
$\boldsymbol{E}_{\text {bio }}$ & Biological reference element for the manufacture of the manufacturing base element \\
$\boldsymbol{E}_{\text {base }}$ & Manufacturing base element \\
$\boldsymbol{E}_{\text {final }}$ & Final manufactured element \\
$\boldsymbol{R}_{\text {bio }}$ & Set of restrictions applied to the biological reference element \\
$\boldsymbol{R}_{\text {base }}$ & Set of restrictions applied to the manufacturing base element \\
$\boldsymbol{D e f}_{\text {bio }}: \boldsymbol{R}_{\text {bio }} \boldsymbol{x} \boldsymbol{E}_{\text {bio }} \rightarrow \boldsymbol{E}_{\text {bio }}$, & Set of deformations to be applied to the biological element \\
$\boldsymbol{D e f}_{\text {base }} \boldsymbol{R}_{\text {base }} \boldsymbol{x} \boldsymbol{E}_{\text {base }} \rightarrow \boldsymbol{E}_{\text {base }}$ & Set of deformations to be applied to the manufacturing base element \\
$\boldsymbol{C}_{\text {bio-base }}$ & Set of quality criteria to be met by the final manufactured element
\end{tabular}

Table 1 specifies each of the parameters to be considered in the model. Meeting the quality criteria $C_{\text {bio-base }}$ requires the application of a set of deformations made to $E_{b i o}$ and $E_{b a s e}$. The set of deformations to be applied to $E_{b i o}$ are described by $D e f_{\text {bio }}$ which remains subject to the biological restrictions defined in the $R_{b i o}$ set and the set of deformations to be made to $E_{\text {base }}$, which are described in $D e f_{\text {base }}$ which in turn remains subject to the restrictions imposed by the $R_{\text {base }}$ set. It is graphically possible to represent the model according to the flow diagram in Fig. 3 .

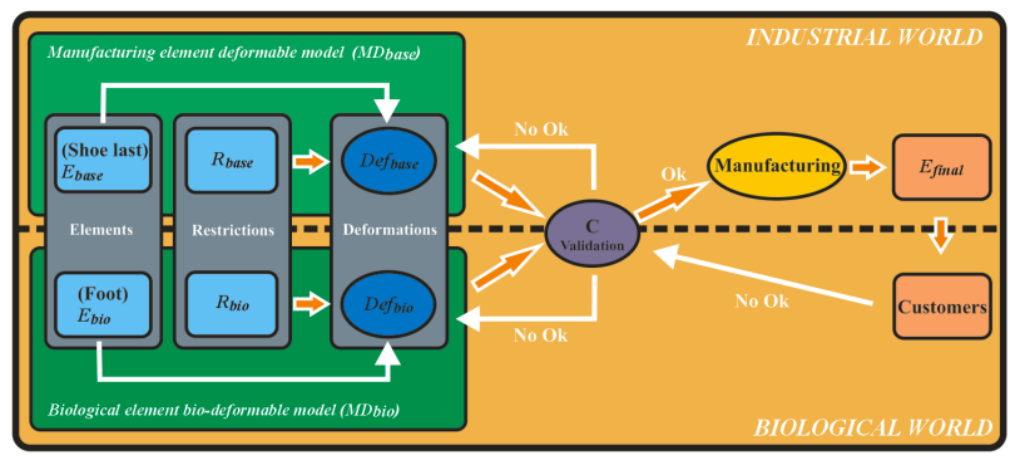

Fig. 3. Model Structure

As a link for all the elements indicated above, two models have been developed, which are dynamically interrelated and are capable of meeting the requirements established in the initial description of the problem. These models became defined as:

- Bio-deformable model, $M D_{\text {bio }}=\left\{E_{b i o}, R_{b i o}\right.$, Def $\left._{\text {bio }}\right\}$.

- Deformable model for the industrial element, $M D_{\text {base }}=\left\{E_{\text {base }}, R_{\text {base }}\right.$, Def $\left.f_{\text {base }}\right\}$.

\subsection{Bio-deformable foot model}

The bio-deformable foot model is defined as: $M D_{b i o}=\left\{E_{b i o}, R_{b i o}, D e f_{b i o}\right\}$. The definition of a series of measurements that characterise the foot is necessary in order to be able to apply deformations to the foot and adapt it to the pitch curve of the last, restricting the foot joint movement in the process. These measurements ${ }^{1}$ are taken as a reference to obtain the anthropometric foot measurements (see Fig. 4).

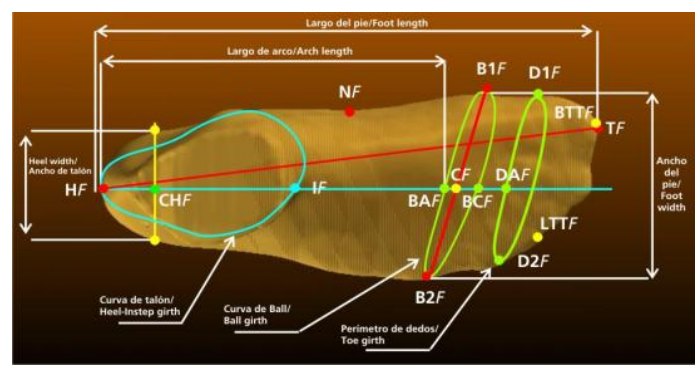

Fig. 4. Characteristic foot measurements

1 The characteristic measurements of both the foot and the last have been defined according to the specifications of the Footwear Technological Institute (Inescop), which establish a series of characteristic points and measurements for feet and lasts that are useful for their application in the manufacture of customised footwear. These specifications are currently being analysed and used in various international projects through the technical standardisation committee ISO TC/137 "Footwear sizing designations and marking systems", with the objective being to convert them into an international standard for footwear so that their formal specification and usefulness are unified and thus can be used by the sector's companies. 
To carry out the deformation of the foot $\left(D e f_{\text {bio }}\right)$, a cage-based deformation method has been used [50]. This technique, widely used for rigging provides a flexible space control of the geometry to be deformed at a relatively low computational cost. The deformation through three-dimensional control cages developed in [50] introduced this new approach which is based on the concept of the "Mean Value Coordinates" (MVC) established by [51] to define the weight functions that govern the deformable space. Initially, Floater introduced the MVCs for two-dimensional polygons as a way to achieve smooth information interpolations in polygons. Later, in [52] the method was expanded, applying it to three-dimensional triangle meshes. Other works appear in the area of automatic deformation control mesh creation: in [53] the semi-automatic creation of skinning templates is proposed for use through these deformation techniques.

Cage-based deformation techniques provide three characteristics that are decisive for their application in customised footwear:

- Speed. Given that the acquisition of the control object should be done quickly and at low computational cost, the amount of interaction of the final user in the foot deformation should be none, with the process being completely automatic. For example, skeleton-based deformation techniques $[54,55,56]$ have, as a main problem, the bone and articulation configuration time by the user.

- Flexibility. The topology of the control object structure should perfectly define the foot articulation points and axes from the morphometric information of the foot. Thus this should allow the dynamic adaptation of the reference foot morphology.

- Adaptability. Later refinements of the model can be easily incorporated through the adequate characterisation of the control cage.

An important aspect of these techniques is the decoupling of the base geometry to be deformed and the control object that manipulates it. These are geometrically different entities, but the control geometry structurally meets the intrinsic characteristics of the base object.

\section{Cage definition}

The foot deformation cage is a control mesh that fits the base object, which is wrapped around and characterised by the cage in such a way that the articulation properties of the object are established. More formally, let a cage $C$ be a polyhedron in $d$ dimensions that is a closed (not necessarily convex) volume with a piecewise linear boundary. In three dimensions, a cage $C$ is a closed region of space bounded by planar faces. Constructing a function that interpolates a set of values defined at vertices of a polygonal mesh is a fundamental operation in computer graphics. Such an interpolant has many uses in applications such as advanced shading, parameterisation and deformation of objects. For closed polygons, MVCs have proven to be an excellent method for constructing such an interpolant. In this paper, we apply MVCs to closed polygonal meshes. Given such a mesh $P$, we show that these MVCs are continuous everywhere and smooth on the interior of $P$. The coordinates are linear on the triangles of $P$ and can reproduce linear functions on the interior of $P$. Given a closed mesh, formed of triangles with vertices $\{p 1, p 2, p 3\}$ and associated intensities $\{f 1, f 2, f 3\}$, the intensity at point $v$ on the interior of the triangle can be expressed in the form [50]:

$$
\hat{f}[v]=\frac{\sum_{j} w_{j} f_{j}}{\sum_{j} w_{j}}
$$

where $w_{j}$ is the area of the triangle $\left\{v, p_{j-1}, p_{j+l}\right\}$. In this formula, note that each weight $w_{j}$ is normalised by the sum of the weights to form an associated coordinate. The interpolant $\hat{f}[v]$ is then simply the sum of the $f_{j}$ times their corresponding coordinate.

Mesh parameterisation methods [57,58,59,60,61] and freeform deformation methods [62,63,64,65] also make heavy use of interpolants of this type. Several papers, [51,66,67,68,69], have focused on building interpolants for nonconvex 2D polygons. In particular, Floater proposed a new type of interpolant based on the "Mean Value Theorem" that generates smooth coordinates for star-shaped polygons. Given a polygon with vertices $p_{j}$ and associated values $f_{j}$, Floater's interpolant defines a set of weight functions $w_{j}$ of the form:

$$
w_{j}=\frac{\tan \left[\frac{\alpha_{j-1}}{2}\right]+\tan \left[\frac{\alpha_{j}}{2}\right]}{\left|p_{j}-v\right|}
$$

where $\alpha_{j}$ is the angle formed by the vector $p_{j}-v$ and $p_{j+l^{-}}-v$. Normalising each weight function $w_{j}$ by the sum of all weight functions yields the mean value coordinates of $v$ with respect to $p_{j}$. In [51] the interpolant was defined from the 
application of the mean value theorem, [70] used a solution to Laplace's equation and [71] was based on the Green functions to obtain their interpolant. In this paper, we opted for the solution described by [51]: Mean Value Coordinates.

\section{Obtaining the deformation cage}

Obtaining the deformation cage is an automatic process based on purely morphological aspects of the user's reference foot. The foot morphology based deformation should be carried out taking the foot's morphometric information into account. It is necessary to obtain a simplified representation that has the following characteristics:

- Anatomical links with the foot. The resulting cage must maintain the anatomical information necessary to carry out the articulation of the foot. This must be based on the characteristic points and curves obtained in the previous section.

- Simplicity. The resulting cage must be as simple as possible, minimising the number of faces as the computational cost of the deformation algorithm application is directly linked to the number of faces in the cage.

- Constraint. The resulting cage must enclose the whole of the reference foot to guarantee its correct articulation.

Below, the process for obtaining an exoskeleton, which defines the articulation points of the foot, is described; this exoskeleton is based on the acquisition of the characteristic planes from the characteristic foot articulation curves. The creation of the deformation cage defines a valid exoskeleton for the representation of the foot in the sagittal-frontal plane. To obtain this cage, we start from the acquisition of some characteristic foot curves that determine the necessary articulation points for the deformation to take place. These curves are (see Fig. 5), $F_{C d 1}$ (metatarsal area), $F_{C d 2}$ (instep are), $F_{C d 3}$ (back heel area) y $F_{C d 4}$ (heel tread area).

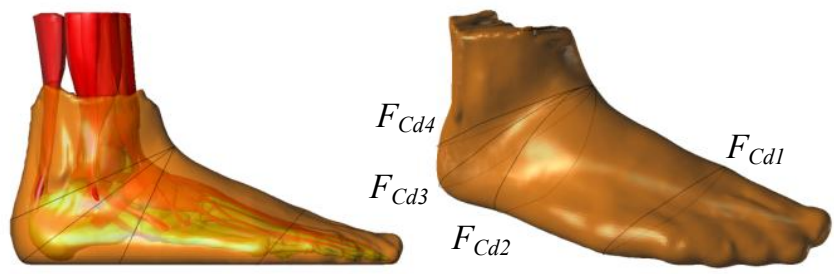

Fig. 5. Characteristic curves for deformation

As can be seen in the previous figure, the curves have direct relations to the foot articulation key areas. These curves are detected automatically through digitisation and their acquisition does not require complex calculations that increase the time needed for the process. Then we proceed with the offset of these characteristic curves $F_{C d 1}, F_{C d 2}, F_{C d 3}$ and $F_{C d 4}$, a $F_{M O f f s e t}$. For this, the following steps are followed:

1. The corresponding planes are obtained which contain the curves $F_{C d l}, F_{C d 2}, F_{C d 3}$ and $F_{C d 4}$. These are: $P F_{C d l}, P F_{C d 2}$, $P F_{C d 3}$ and $P F_{C d 4}$.

2. The planes $P F_{C d 1}, P F_{C d 2}, P F_{C d 3}$ and $P F_{C d 4}$ are intersected with $F_{M O f f s e t}$, obtaining the curves $F_{C d o f f s e t 1}, F_{C d o f f s e t 2}, F_{C d o f f s e t 3}$ and $F_{C d o f f s e t}$.

Finally, the bounding boxes for each of the curves $F_{C d o f f s e t}, F_{C d o f f s e t 2}, F_{C d o f f s e t 3}$ and $F_{C d o f f s e t}$ are obtained, and each of the corresponding vertices are triangulated, thus obtaining the polygonal mesh $F_{\text {DefCage }}$ (see Fig. 6).
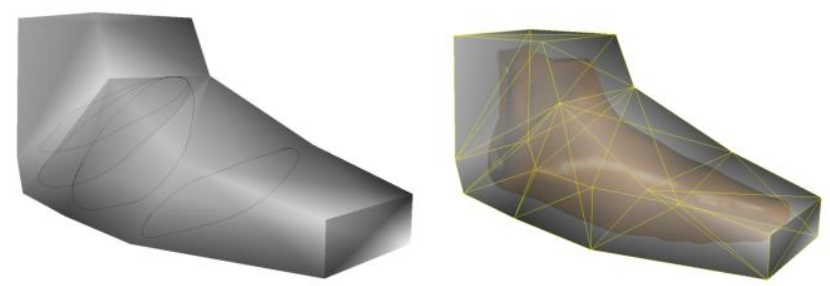

Fig. 6. Exoskeleton $F_{\text {DEFcage }}$ for foot deformation

In this way, the foot exoskeleton $F_{D E F c a g e}$ has been obtained with articulation properties. The foot deformation consists of the rotation of the exoskeleton vertices about the foot's anatomical rotational axes. The described method has the following advantages:

1.Homeomorphism. The automatic method is able to obtain a homeomorphic cage with regard to the original geometry, the foot.

2. Shape preservation. Dependant on the base curves.

3. Automatic. The method is totally automatic and does not need user interaction, except for some minor adjustment parameters. The user does not manipulate the vertices of the resulting cage at any time.

4.Efficiency. Due to it being an automatic method the resulting cage can be obtained in seconds. 
5.Morphology. The acquisition of the cage is completed through the inclusion of parameters related to the morphology of the original object, including all information referring to the anatomy of the foot (see section on foot points and measurements).

6. Expandable. It can be seen that the method is expandable for the modelling and deformation of other types of base geometries, from which anatomical information can be obtained.

7. Generality. Obtaining the cage does not solely apply to polygonal meshes, but it can also be extrapolated to different 3D geometric entities, point clouds (with or without normal orientation), NURBS surfaces, curve groups, etc.

\section{Applying the deformation cage}

The foot deformation is achieved through the completion of the necessary transformations to be applied to the deformation cage. For this, first the foot sole curve $F_{C p}$ must be deformed, in order to minimise the error in the calculation of the distance between the last pitch curve $L_{C q}$ and the foot sole curve $F_{C p}$ (see Fig. 7).
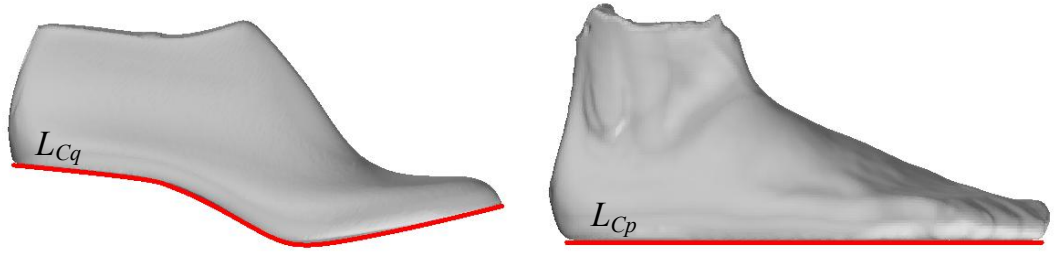

Fig. 7. Last pitch $\mathrm{L}_{\mathrm{Cq}}$ and foot sole $\mathrm{F}_{\mathrm{Cp}}$ curves

The adjustment of the variables that intervene in the foot deformation process implies the finding of three rotation values $\left(R_{1}, R_{2}\right.$ and $\left.R_{3}\right)$ and a translation $(T)$. For this, the minimisation of an objective function defined in Eq. (3), (4), (5) is carried out through the conjugate gradient method [72], which provides a valid solution in a time that can be assumed as an indispensible efficiency requirement for the process. A similar approach is completed in the work of [73] in which the minimisation of an objective function is completed through the conjugate gradient method to obtain a better suitability of a set of points on the last surface. The objective function to be minimised is defined as:

$$
\operatorname{Min} F(x)=\operatorname{Dist}\left(L_{C q}, F_{C p}, R, T, R e s\right)
$$

where $R$ is defined as the sum of the 3 following rotations:

$$
R=\cup(R i), i=1,2,3
$$

considering the restriction Res as:

$$
\operatorname{Res}=(H L-F L=B D L)
$$

with $\mathrm{BD} L$ being the minimum necessary distance between the furthest back points of the last and foot to allow the correct in-shoe mobility of the foot.

The rotations $R_{i}$ are finally applied as indicated below:

- $R_{l}$. Rotation of rear-metatarsal vertices. Axis defined by a metatarsophalangeal joint. The axis MetFalAxis $=\vec{v}$. The rotational angle of the vertices to be applied in this rotation is defined as $\theta_{l}$ (see Fig. 8-10). The set of restrictions defined in $R_{b i o}$ provides the maximum angle to be assigned to $\theta_{l}$.

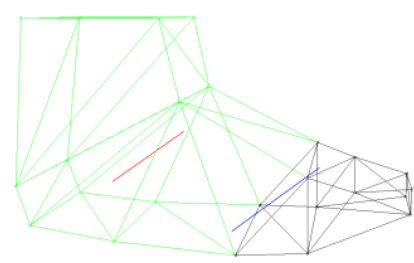

Fig. 8. Metatarsal-rear deformation area

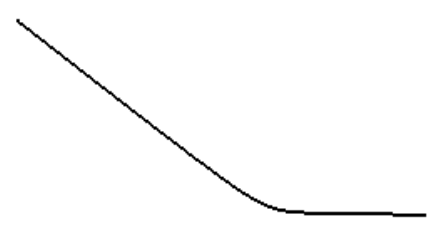

Fig. 9. Rear-metatarsal deformation of $F_{C p}$

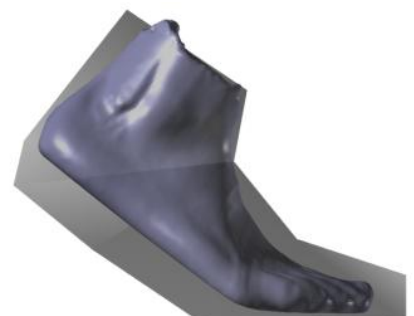

Fig. 10. Step 1: Deformation of foot flexion

- $R_{2}$. Rotation of heel vertices. Axis defined by the Chopart joint. The axis ChopAxis $=\vec{w}$. The rotational angle of the vertices to be applied in this rotation is defined as $\theta_{2}$ (see Fig. 11-13). The set of restrictions defined in $R_{b i o}$ provides the maximum angle to be assigned to $\theta_{2}$. 


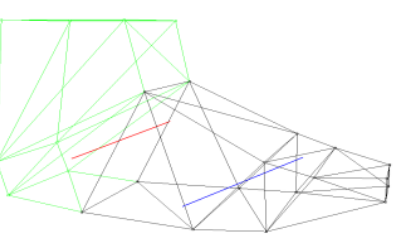

Fig. 11. Heel deformation area

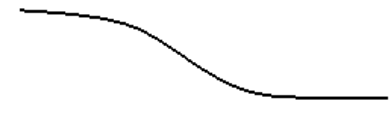

Fig. 12. Heel deformation of $F_{C p}$

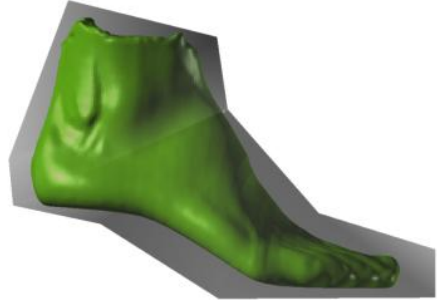

Fig. 13. Step 2: Deformation of heel flexion

- $R_{3}$. Front-metatarsal vertices rotation. Axis defined by a metatarsophalangeal joint. The axis MetFalAxis $=\vec{v}$. The rotational angle of the vertices to be applied in this rotation is defined as $\theta_{3}$ (see Fig. 14-16). The set of restrictions defined in $R_{b i o}$ provides the maximum angle to be assigned to $\theta_{3}$.

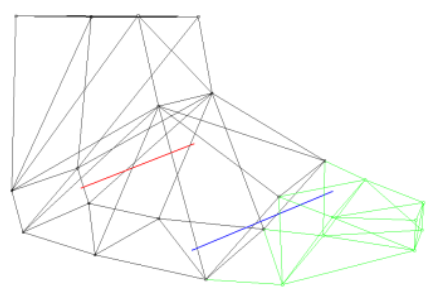

Fig. 14. Front-metatarsal deformation area

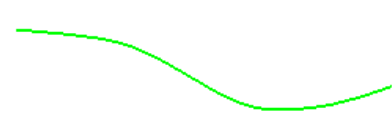

Fig. 15. Front-metatarsal deformation of $F_{C p}$

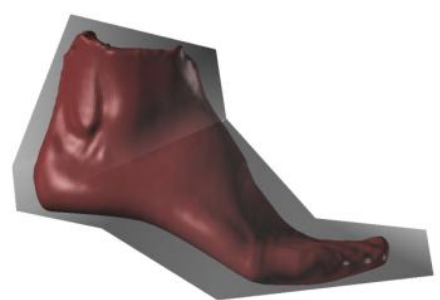

Fig. 16. Step 3: Deformation of toe flexion

For each of the axes:

- Axis MetFalAxis ( $\vec{v})$ : Defined by the B1F and B2F points.

- Axis ChopAxis ( $\vec{w}$ ): Defined by the $\mathrm{N} F$ point. Vector perpendicular to the $X Z$ plane (see Fig. 17).
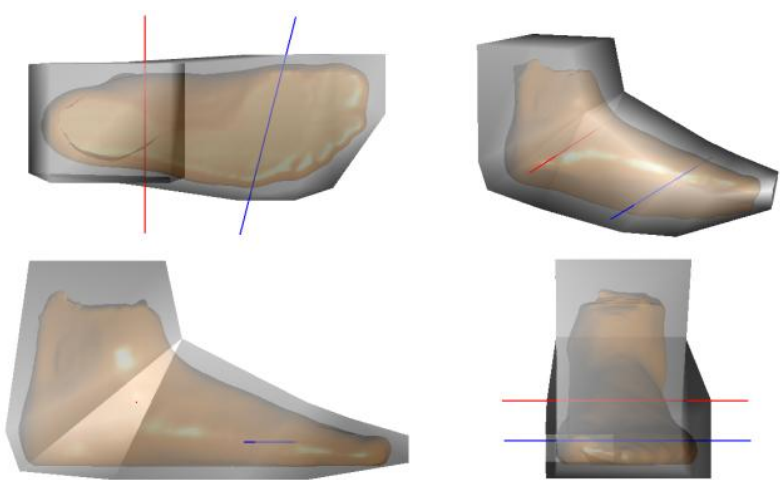

Fig. 17. Rotation axes. In blue MetFalAxis. In red ChopAxis

Finally, the translation $T$ is defined as the translation of the deformed curve $F_{C p}$ in the $X Z$ plane, through the $\vec{t}$ vector (see Fig. 18).

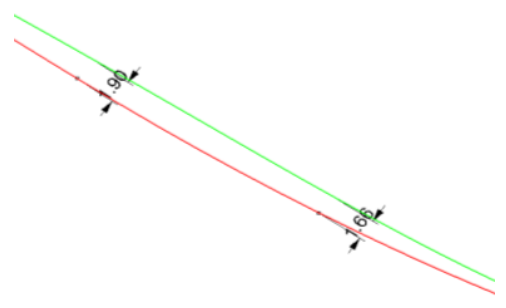

Fig. 18. Translation of $\mathrm{F}_{\mathrm{Cp}}$ and error calculation

Once the variables of the problem, the rotation angles $\left(\theta_{1}, \theta_{2}, \theta_{3}\right)$ and the translation vector $(\vec{t})$ that minimise the distance between the curves have been obtained, these transformations are applied to the foot mesh $F_{M}$. Once the transformations have been applied, the foot is deformed, represented by the polygonal mesh $F_{\text {MDef }}$ 


\section{Foot measurements and deformation error}

Table 2 shows the deviation incurred in the values obtained after deformation of the foot. These measurements are compared to measurements taken from the scans of the four foot geometry which has been digitized with different heights, using a support element in the heel area in order to obtain 40,60 and $80 \mathrm{~mm}$ in height. Deformed foot measures are presented with nomenclature Foot $X(D)$ - XXmm.

Table 2

Comparison of foot, different high heel and deformed foot measurements (in mm)

\begin{tabular}{|c|c|c|c|c|c|}
\hline & Foot Length & Foot Width & Heel Width & $\begin{array}{c}\text { Medium } \\
\text { Instep Girth }\end{array}$ & $\begin{array}{c}\text { Standard Ball } \\
\text { Girth }\end{array}$ \\
\hline Foot 1 - Flat & 237,30 & 88,90 & 66,00 & 233,40 & 225,30 \\
\hline Foot $1-40 \mathrm{~mm}$ Heel & 245,00 & 84,40 & 64,60 & 237,20 & 220,30 \\
\hline Foot 1(D) - 40mm Heel & 240,20 & 88,00 & 65,50 & 235,20 & 224,50 \\
\hline Error Deviation & $2 \%$ & $4 \%$ & $1 \%$ & $1 \%$ & $2 \%$ \\
\hline Foot 1 - 60mm Heel & 241,20 & 85,50 & 66,90 & 236,20 & 222,90 \\
\hline Foot 1(D) - 60mm Heel & 241,50 & 88,20 & 65,80 & 236,40 & 225,30 \\
\hline Error Deviation & $0 \%$ & $3 \%$ & $2 \%$ & $0 \%$ & $1 \%$ \\
\hline Foot $1-80 \mathrm{~mm}$ Heel & 245,90 & 84,50 & 65,00 & 233,00 & 222,10 \\
\hline Foot 1(D) - 80mm Heel & 241,80 & 88,00 & 65,20 & 234,50 & 224,60 \\
\hline Error Deviation & $2 \%$ & $4 \%$ & 0\% & $1 \%$ & $1 \%$ \\
\hline Foot 2 - Flat & 246,50 & 93,30 & 66,80 & 233,90 & 230,10 \\
\hline Foot 2 - 40mm Heel & 254,80 & 91,40 & 68,00 & 234,20 & 228,30 \\
\hline Foot 2(D) - 40mm Heel & 248,10 & 92,50 & 65,30 & 234,30 & 229,30 \\
\hline Error Deviation & $3 \%$ & $1 \%$ & $4 \%$ & $0 \%$ & $0 \%$ \\
\hline Foot $2-60 \mathrm{~mm}$ Heel & 252,50 & 89,70 & 65,80 & 232,10 & 222,80 \\
\hline Foot 2(D) - 60mm Heel & 249,80 & 92,40 & 65,40 & 234,50 & 228,40 \\
\hline Error Deviation & $1 \%$ & $3 \%$ & $1 \%$ & $1 \%$ & $3 \%$ \\
\hline Foot $2-80 \mathrm{~mm}$ Heel & 256,40 & 89,10 & 65,90 & 229,60 & 223,00 \\
\hline Foot 2(D) - 80mm Heel & 249,90 & 92,00 & 65,80 & 233,80 & 228,10 \\
\hline Error Deviation & $3 \%$ & $3 \%$ & $0 \%$ & $2 \%$ & $2 \%$ \\
\hline Foot 3 - Flat & 221,00 & 89,60 & 65,40 & 222,70 & 222,00 \\
\hline Foot 3 - 40mm Heel & 227,60 & 88,20 & 63,20 & 218,50 & 220,90 \\
\hline Foot 3(D) - 40mm Heel & 223,10 & 89,00 & 65,20 & 223,50 & 221,30 \\
\hline Error Deviation & $2 \%$ & $1 \%$ & $3 \%$ & $2 \%$ & $0 \%$ \\
\hline Foot $3-60 \mathrm{~mm}$ Heel & 223,40 & 86,20 & 62,30 & 216,00 & 218,70 \\
\hline Foot 3(D) - 60mm Heel & 223,60 & 89,20 & 65,20 & 222,80 & 220,40 \\
\hline Error Deviation & $0 \%$ & $3 \%$ & $5 \%$ & $3 \%$ & $1 \%$ \\
\hline Foot 3 - 80mm Heel & 227,80 & 83,90 & 61,10 & 215,60 & 214,60 \\
\hline Foot 3(D) - 80mm Heel & 224,10 & 88,40 & 64,20 & 222,30 & 221,60 \\
\hline Error Deviation & $2 \%$ & $5 \%$ & $5 \%$ & $3 \%$ & $3 \%$ \\
\hline Foot 4 - Flat & 242,00 & 93,20 & 75,40 & 229,00 & 229,00 \\
\hline Foot 4 - 40mm Heel & 247,50 & 90,20 & 72,70 & 229,30 & 225,60 \\
\hline Foot 4(D) - 40mm Heel & 243,60 & 92,10 & 74,80 & 228,50 & 228,50 \\
\hline Error Deviation & $2 \%$ & $2 \%$ & $3 \%$ & $0 \%$ & $1 \%$ \\
\hline Foot $4-60 \mathrm{~mm}$ Heel & 248,50 & 88,20 & 75,40 & 229,30 & 223,30 \\
\hline Foot 4(D) - 60mm Heel & 244,90 & 92,20 & 74,60 & 228,60 & 228,20 \\
\hline Error Deviation & $1 \%$ & $5 \%$ & $1 \%$ & $\mathbf{0 \%}$ & $2 \%$ \\
\hline Foot 4 - 80mm Heel & 250,70 & 88,90 & 74,50 & 225,10 & 225,60 \\
\hline Foot 4(D) - 80mm Heel & 244,50 & 92,20 & 74,25 & 228,60 & 228,30 \\
\hline Error Deviation & $2 \%$ & $4 \%$ & $0 \%$ & $2 \%$ & $1 \%$ \\
\hline Error Deviation Average & $1 \%$ & $3 \%$ & $2 \%$ & $1 \%$ & $1 \%$ \\
\hline
\end{tabular}




\subsection{Deformable last model}

Once the bio-deformable foot model has been defined, a deformable last model is defined taking into account the set of restrictions that must be met during the deformation (modification) process of the last, restrictions that are defined by the previously deformed reference foot. The interconnection between both models allows an effective comparison between different entities, foot and last, in order to carry out the ideal modifications on the last based on the structure and morphology of the reference foot. The parameterisation of the last, defined in $E_{\text {base }}$ (see Fig. 19), provides the necessary information (measurements) to begin the comparison with the reference foot and the application of the suitable modifications.

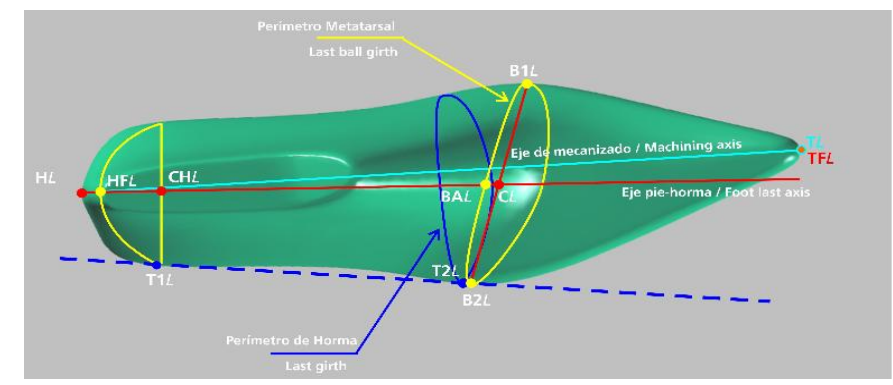

Fig. 19. Last model $\boldsymbol{E}_{\text {base }}$

There are many studies based on sketching techniques for the completion of volumetric deformation of threedimensional objects. The objects are mainly characterised by the modification of a three-dimensional object through a specific interface to obtain a specific result. The works based on these techniques allow these surfaces to be doubled [74,75,76,77,78], and twisted [79], silhouettes to be modified [80,81], segmentation [76,82], free deformation [83] and finally have the affine transformations applied [84]. There are also works in which character animation is carried out by this type of technique [75]. In the case of [83] articulation points are extracted through image processing techniques such as erosion, applying physical and geometric constraints to avoid invalid poses. In the work by [84] the deformation is applied through curves defined in orthogonal planes that are modified manually to apply the appropriate deformation. The initial curves are also defined manually before making deformations. In this paper, techniques are used that are inspired by those described by [85] modifying certain aspects to make them more suited to the modifications to be made to the last. The main contribution comes from the method used to relate deformation curves and the interpolation function used to define the field of action. The parametric curves that characterise the area of the last to be deformed are previously defined through the acquisition of characteristic curves that parametrically define the base last. These areas are those that should be locally modified using control curves. This work introduces the concept of a network of deformation curves, as determined deformations should be made using a set of control curves or, as they are called in this paper, a Network of Deformation Control Curves $\left(\mathrm{NdC} \mathrm{C}^{2}\right)$.

\section{Network of deformation control curves $\left(\mathrm{NdC} \mathrm{C}^{2}\right)$}

Each of the curves belonging to the $N d C^{2}$ deforms an area of the surface. We define the $N d C^{2}$ system $=\{D c, R c, s, r, f$, $\left.C_{M}\right\}$, where $N$ is the total number of deformation curves in the $N d C^{2} ; D c_{i}$ and $R c_{i}$ are free-form parametric curves, $s_{i}$ is a scalar that controls radial scaling around the curve, $r_{i}$ is a scalar value defining a radius of influence around the reference curve and $C_{M}$ is the method of correspondence between any point defined in $R c_{i}$ and $D c_{i}$; and the scalar function $f: \mathfrak{R}^{+} \rightarrow[0,1]$ is to preserve the continuity in the deformation according to the radius of influence $r_{i}$. The function $f$ shows continuity $C^{l}$ and is monotonically-decreasing with $f(0)=1, f(x)=0$ for $x \geq 1$ and $f^{\prime}(0)=0, f^{\prime}(1)=0$. In this paper, unlike [86], $f$ is the Gaussian function represented as:

$$
f(x)=\frac{1}{\sqrt{2 \pi \sigma}} e^{\frac{-(\mu-x)^{2}}{2 \sigma^{2}}}
$$

where $\mu$ and $\sigma$ are real positive constants, which control the origin point and the width of the bell respectively.

The parameter $r_{i}$ defines, for the $i$-nth control curve, the reach of the deformation on the original object, controlling and guaranteeing the continuity of the deformation through the $f$ parameter. Together with the scaling parameter $s_{i}$, and given $r_{i}$ and $f$, a control curve is defined by means of the specification of the original curve $R c_{i}$ and the copy of the curve $D c_{i}$, in which the shape will be altered to carry out the controlled deformation of the geometry. Each control curve, $R c_{i}$ specifies a tube-shaped surface around the curve of $r_{i}$, radius, the deformation influence for points of the object within this surface that is controlled by the density function $f$. The deformation at a point $P$ is related to the 
deviation of the closest point on the reference curve $R c_{i}\left(p_{R}\right)$ from a corresponding point on the deformation curve $D c_{i}$. We use a direct correspondence between curves $R c_{i}$ and $D c_{i}$ based on parameter value (parametric deformation). The correspondence used can be directly varied as a method parameter, defining the three possibilities shown in Fig. 20-22:

- Closest. Let $C_{1}\left(u_{1}\right) \in R c$ and $C_{2}\left(u_{2}\right) \in D c$ be the curves defined in the same domain by $u_{1,2} \in[0,1]$. For any point $P \in \mathfrak{R}^{3}$ on $C_{1}$, let $p_{c 2} \in[0,1]$ be the parameter value that minimises the Euclidean distance between point $P$ and curve $C_{2}(u)$. If there is more than one minimum, we define $p_{c 2}$ to be the parameter with the smallest value.

$$
p_{c 2} \in[0,1] / \forall u_{2} \in[0,1] ;\left|C_{2}\left(p_{c 2}\right)-P\right| \leq\left|C_{2}\left(u_{2}\right)-P\right|
$$

- Parametric. Let $C_{1}\left(u_{1}\right) \in R c$ and $C_{2}\left(u_{2}\right) \in D c$ be the curves defined in the same domain by $u_{1,2} \in[0,1]$. For any point $P \in \mathfrak{R}^{3}$ on $C_{1}$, let $p_{c 2} \in[0,1]$ be the parameter value that equals the parameter value of point $P$ on $C_{l}$ and curve $C_{2}(u)$.

$$
p_{c 2}=u_{2} / C_{2}\left(u_{2}\right)=P ; P=C_{1}\left(u_{1}\right) ; u_{1}, u_{2} \in[0,1]
$$

- Tangent distance. Let $C_{1}\left(u_{1}\right) \in R c$ and $C_{2}\left(u_{2}\right) \in D c$ be the curves defined in the same domain by $u_{1,2} \in[0,1]$ and let the vector $\vec{n}$ be the normal at point $P \in \mathfrak{R}^{3}$ on $C_{l}$, we define $p_{c 2} \in[0,1]$ to be the parameter resulting from the intersection of the line generated with vector $\vec{n}$ and curve $C_{2}(u)$. If there is more than one intersection, we define $p_{c 2}$ to be the parameter at the closest distance from curve $C_{l}$.

$$
\begin{gathered}
p_{c 2}=\text { Intersection }\left(C_{2}\left(u_{2}\right), \text { Straight line }\left(\operatorname{Normal}\left(C_{1}\left(u_{1}\right)\right), P\right) ;\right. \\
P^{\prime}=C_{2}\left(p_{c 2}\right), P=C_{1}\left(u_{1}\right) ; u_{1}, u_{2} \in[0,1]
\end{gathered}
$$

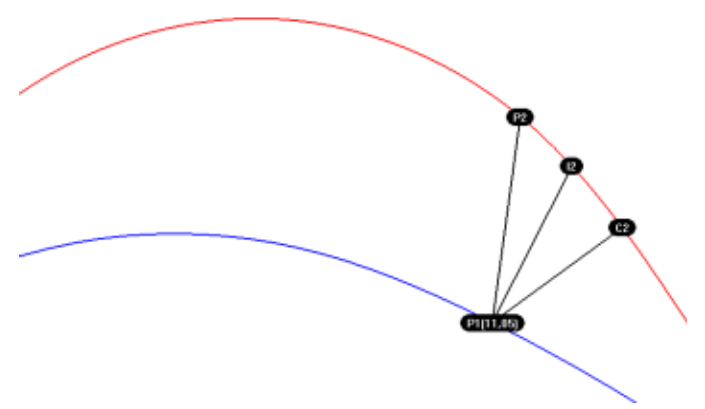

Fig. 20. P2: parametric, C2: proximity, I2: tangency

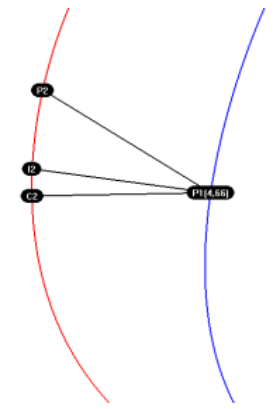

Fig. 21. $\mathrm{P} 2$ : parametric, $\mathrm{C} 2$ : proximity, I2: tangency

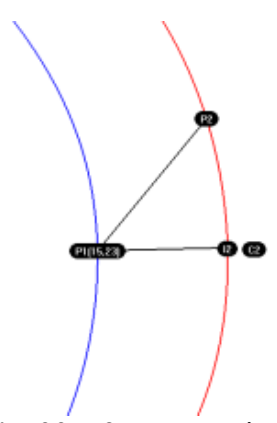

Fig. 22. P2: parametric, $\mathrm{C} 2$ : proximity, I2: tangency

For any point $P$ and curve $R c_{i}$, we define the function $F\left(P, R c_{i}\right)$ as:

$$
F\left(P, R c_{i}\right)=f\left(\frac{\left\|P-R c_{i}\left(p_{C}\right)\right\|}{r_{i}}\right)
$$

From the properties of $f$ it is clear that $F\left(P, R c_{i}\right)$ varies from zero for $\left\|P-\mathrm{R} c_{i}\left(p_{C}\right)\right\| \geq r_{i}$ (points outside the offset volume defined by $R c_{i}$ and $r_{i}$ ), to $F\left(P, R c_{i}\right)=1$ when $\left\|P-R c_{i}\left(p_{C}\right)\right\|=0$ ( $P$ lies on $C$ ). $F\left(P, R c_{i}\right)$ defines the influence that a curve $R c_{i}$ has on a point $P$.

As with any deformation, this kind of deformation is a point-wise function mapping $\mathfrak{R}^{3}$ onto $\mathfrak{R}^{3}$. For each object $O$, let $P_{O}$ be the point-based representation to which the deformation will be applied. $P_{O}$ contains all points necessary to construct or approximate an object's surface. This method allows the application to any type of geometric entity, such as: NURBS surfaces and curves, polygonal meshes or point clouds. When an object $O$ is bound to a $N d C^{2}$, the parameters $p_{\mathrm{Ri}}$ and $F\left(P, R c_{i}\right)$ are computed for every point $P \in P_{O}$. Only points on the object within the offset volume of radius $r_{i}$ from the curve $R c_{i}$ will be deformed. When $D c_{i}$ is manipulated and its geometry is altered, the object is deformed for every point $P$ for which $F\left(P, R c_{j}\right)>0$ :

1. Uniformly scale $P$ about point $R c_{i}\left(p_{R}\right)$ resulting in point $P_{s}$. Specifically, $P_{s}=P+\left(P-R c_{i}\left(p_{R}\right)\right) \cdot\left(1+\left(s_{i}-1\right) \cdot F\left(P, R c_{i}\right)\right)$.

2. Let $C^{\prime}(u)$ be the tangent vector to curve $C$ at $u$, and let $\theta_{i}$ be the angle between $D c_{i}{ }^{\prime}\left(p_{R}\right)$ and $R c_{i}{ }^{\prime}\left(p_{R}\right)$. Rotate $P_{s}$ by the angle $\theta_{i} F\left(P, R c_{i}\right)$, around the axis $D c_{i}{ }^{\prime}\left(p_{R}\right) \cdot R c_{i}{ }^{\prime}\left(p_{R}\right)$, about point $R c_{i}\left(p_{R}\right)$. This results in point $P_{r}$. 
3. Finally add the translation $\left(D c_{i}\left(p_{R}\right)-R c_{i}\left(p_{R}\right)\right) \cdot F\left(P, R c_{i}\right)$. The resulting deformed point, $P_{\text {def }}$, is thus $P_{\text {def }}=P_{r}+\left(D c_{i}\left(p_{R}\right)-\right.$ $\left.R c_{i}\left(p_{R}\right)\right) \cdot F\left(P, R c_{i}\right)$.

This deformation method shows the following properties:

- Objects are not deformed initially, after the creation of the copies of the deformation curves, both $R c_{i}$ and $D c_{i}$ coincide with each other.

- Points on the object outside the offset volume of radius $r_{i}$ from the reference curve (points $P$ with $\left.F\left(P, R c_{i}\right)=0\right)$ are not deformed regardless of the value of $s_{i}$.

- Points on the object that are on the reference curve, when the object is bound to the network of curves, track the deformation precisely as they are directly affected by the reference curve and the implied deformation.

- The deformation of points on the object between those on the reference curve and those outside its realm of influence is smooth and intuitive. The factor $F\left(P, R c_{i}\right)$ controls the attenuation of the deformation, varying from precise tracking for points on the reference curve to no deformation at or beyond the offset volume boundary.

\section{Deformation curves}

We use several deformation curves to modify the last. This kind of final deformation is carried out by blending each of the deformations applied by each of the deformation curves from the network. Let a network of deformation curves be defined as $N d C^{2}$ and let $P$ be a point on the object to be deformed, we define $P_{d e f i}$ as the point resulting from the deformation applied by curve $D c_{i}$. Let $\Delta P_{i}=P_{\text {defi }}-P$. The deformed point $P_{\text {def }}$ resulting from the blend of all deformation curves is defined as follows:

$$
P_{\text {def }}=P+\frac{\sum_{i=1}^{n} \Delta P_{i} \cdot\left\|\Delta P_{i}\right\|^{m}}{\sum_{i=1}^{n}\left\|\Delta P_{i}\right\|^{m}}
$$

The resulting behaviour varies with $m$, from a single average of the $\Delta P_{i}$ when $m=0$, converging to $\max \left\{\Delta P_{i j}\right\}$ for large $m$. For better control over the network of deformation curves, each curve should have its own exponent $m_{i}$, giving finer control over its contribution to the final deformation result.

$$
P_{\text {def }}=P+\frac{\sum_{i=1}^{n} \Delta P_{i} \cdot\left\|\Delta P_{i}\right\|^{m_{i}}}{\sum_{i=1}^{n}\left\|\Delta P_{i}\right\|^{m_{i}}}
$$

Also, a parameter similar to $m$ is introduced in the above formula which is based on the distance from the deformation curve with regard to the point to be deformed. Depending on the proximity of the point to the deformation curve, its behaviour is modelled through $F\left(P, R c_{i}\right)$ controlling its proximity. The formula used is as follows:

$$
P_{\text {def }}=P+\frac{\sum_{i=1}^{n} \Delta P_{i} \cdot F\left(P, R c_{i}\right)^{k}}{\sum_{i=1}^{n} F\left(P, R c_{i}\right)^{k}}
$$

The factor $k$ has a similar effect to $m$, and can be combined with all those parameters defined earlier.

\section{Basic last modifications}

These basic modifications are carried out for the particular treatment of the critical areas on the last in order to guarantee the fit with regards to the original foot. These basic modifications do not completely resolve severe problems for feet with marked deformities. The deformation technique used is based on the method described in the previous section, $N d C^{2}$, with the number of control curves used in $D c$ and $R c$ depending on the type of modification to be made. The modified last is called $L_{S D e f}$. Some of the most commonly used techniques for last modification are shown in Fig. 23: 


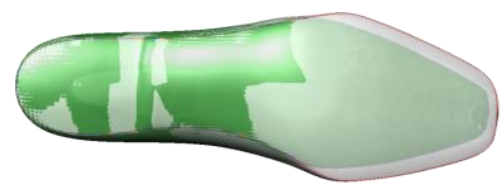

Sole

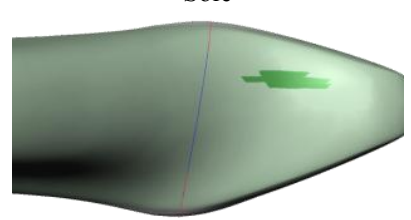

Anatomic ball

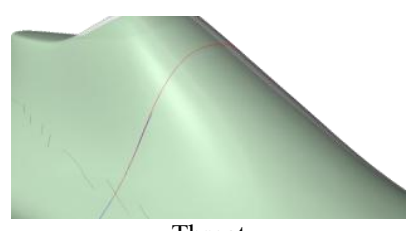

Throat

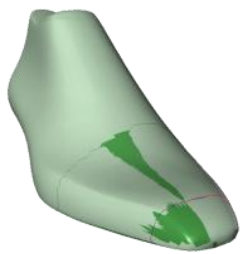

First toe

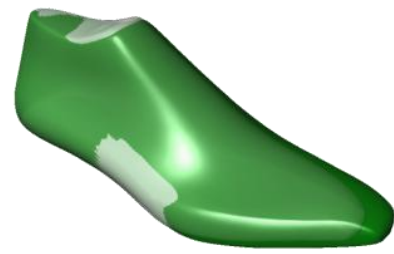

Localised enlargement

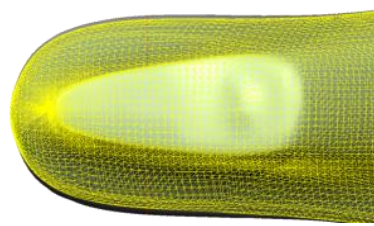

Heel Width

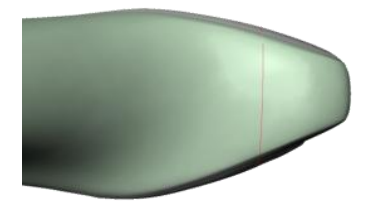

Toes

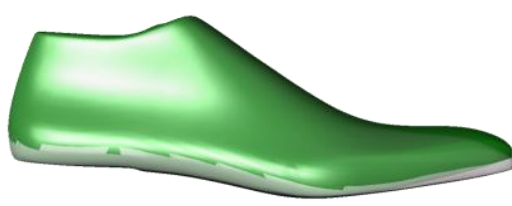

Pitch

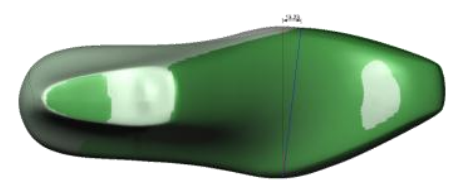

Metatarsal repositioning

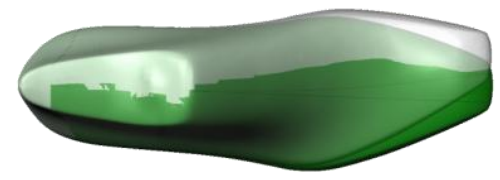

Last rotation

Fig. 23. Common last modifications

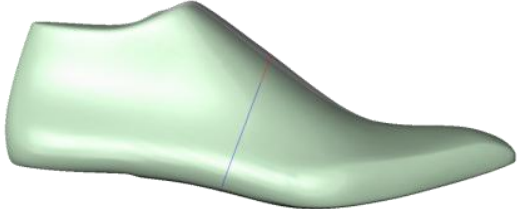

Medium instep

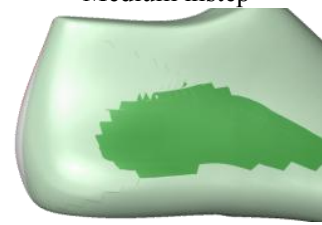

Heel curve

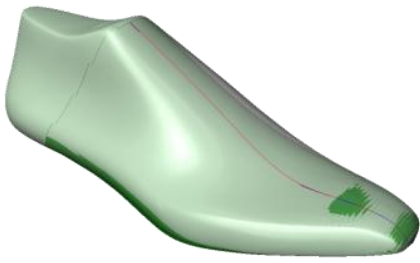

Instep

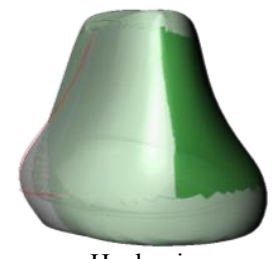

Heel pain

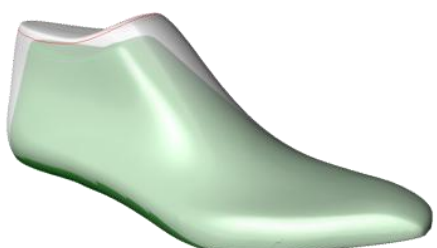

Foot-leg joint

\section{Experimental}

A practical case is presented in which the customisation of the last is carried out for a reference foot with a severe metatarsalgia. The user has a very notable pathology (metatarsalgia, hallux-valgus) in both feet, which causes severe discomfort when using a standard pair of shoes. The patient is an adult female aged 55.

Step 1. Selecting the footwear style.

As a first step in this practical case of customisation, the base footwear style is selected, which provides the base last on which the ideal modifications are to be made (see Fig. 24). In this case, the model has a low heel height.

Step 2. Digitising the user's feet.

Then the reference (right) foot is digitised, which will be compared to the original last which corresponds to the chosen style (see Fig. 24).
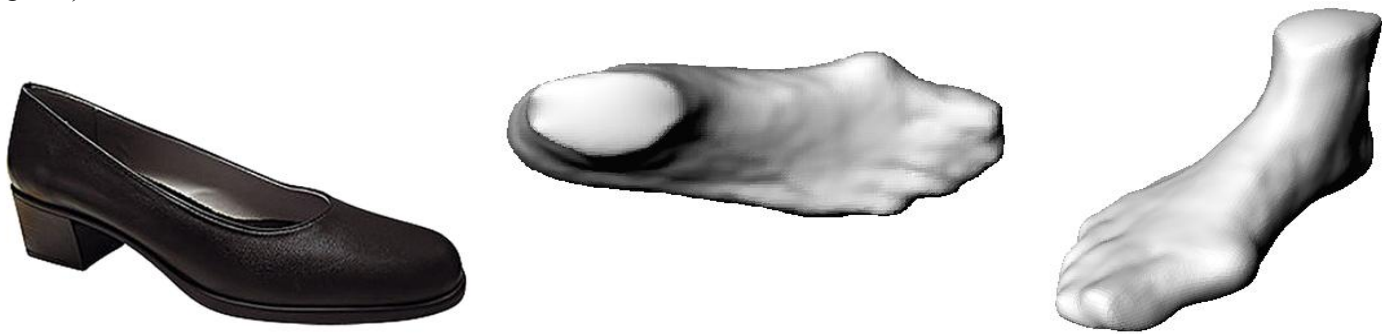

Fig. 24. Footwear style and reference foot 
Step 3. Obtaining the original last and initial foot-last positioning.

The original last is obtained from the chosen footwear style. This last has been adequately graded to ensure that its effective length corresponds to the length of the reference foot. This last will be modified considering the measurements obtained from the reference foot (see Fig. 25).

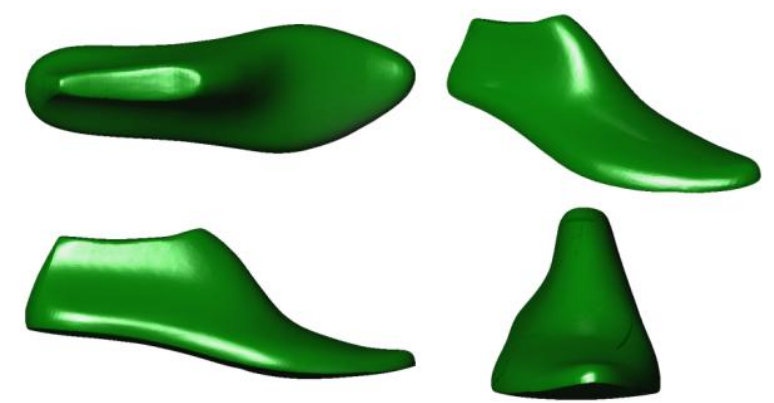

Fig. 25. Original last

The original last and the reference foot are positioned adequately as a prior step to the measuring process. This positioning directly influences the customisation quality (see Fig. 26). In our case, due to the pronounced last pitch, the two geometries are positioned in such a way that the foot metatarsals are aligned with the last's most prominent points.

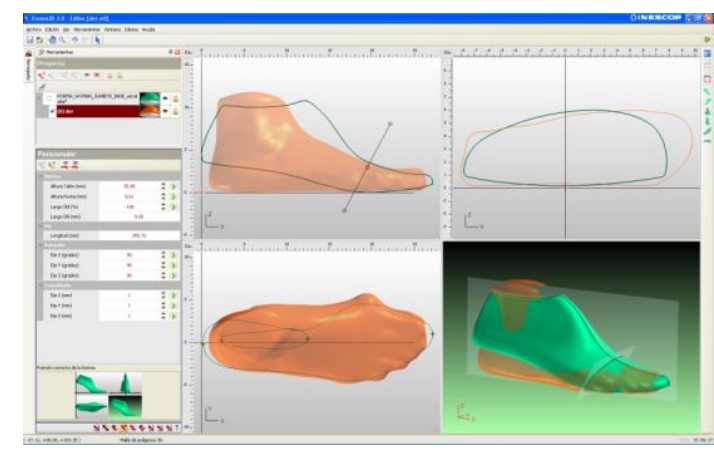

Fig. 26. Initial foot-last positioning

Step 4. Deforming the foot.

The foot deformation process is then started, obtaining the corresponding deformation cage (see Fig. 27).

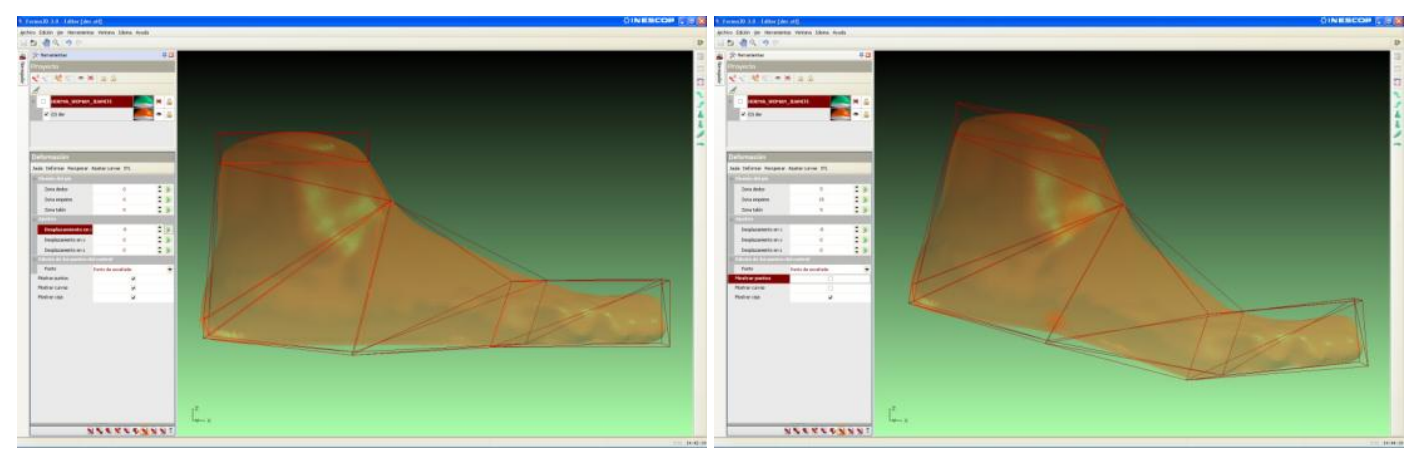

Fig. 27. Obtaining the foot deformation cage and the deformed foot

The parameters, corresponding to the deformation that defines the best possible fit with regard to the last pitch curve, are calculated. These have the following values:

1. Rot 1: Metatarsophalangeal joint axis $\rightarrow$ toe area $=3^{\circ}$ rotation

2.Rot 2: Metatarsophalangeal joint axis $\rightarrow$ instep area $=15^{\circ}$ rotation

3. Rot 3: Chopart joint axis $\rightarrow$ Heel area $=5^{\circ}$ rotation

Step 5. Measuring and comparing the original last.

The measurement and comparison of measurements between the deformed foot and the original last is completed in this step, as a base for the decisions to be made for the modification of the last (see Fig. 28). 


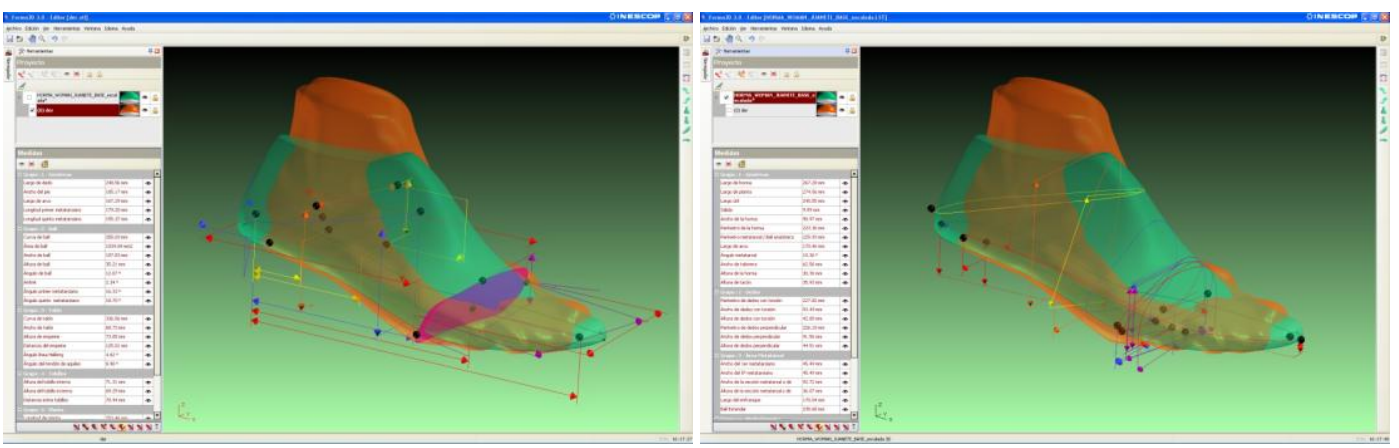

Fig. 28. Foot and last measurements

Step 6. Modifying the last.

After the analysis of the measurements obtained for the deformed foot and the original last, the ideal modifications are then made on the last. This is carried out in the following manner through the $N d C^{2}$ method:

1.Last pitch modification. It is essential that last is supported on the SP $L$ point (see Fig. 19), situated at $69 \%$ of the last effective length. The original last was not correctly supported on this point due to an alteration of the metatarsal position and an incorrect definition of this position.

2. Metatarsal area modification. The ball girth is increased by $13 \mathrm{~mm}$ to provide space for the $5^{\text {th }}$ metatarsal, which is very prominent due to a metatarsalgia (hallux-valgus). The increase requires that the width of the ball curve is increased more for the medial side than the lateral side, as the deformation of the foot is more marked on this side than the other.

3. Heel area modification. It is increased by $3 \mathrm{~mm}$ on both sides from the most prominent points of the heel curve. The upper part of the heel curve is left unaltered, as this should conveniently hold the foot tightly at this height, and the selected footwear style is a court shoe, which has a low profile in this area.

4. Modification of the medium instep area. The medial side is reduced to be a bit tighter; the width is increased by 12 $\mathrm{mm}$ on both sides, with higher intensity in the medial side than the lateral side.

5.First toe area modification. The first toe height is increased by $10 \mathrm{~mm}$, as this toe needs more height on average than the others. This deformation implies an aesthetic modification of the last.

6. Aesthetic toe modification (I). An enlargement of the toe and an aesthetic re-design are carried out to compensate for the modification made to the metatarsals.

7. Aesthetic toe modification (II). The toe slope is modified to give it a better appearance. Having enlarged the toe, it is possible to reduce the gradient of the last slope and thus improve the general appearance of the last

8. Ball curve adjustment. A curve girth of $240 \mathrm{~mm}$ is to be achieved.

The network of deformation curves used to carry out these modifications is shown in Figs. 29-30. In blue, the origin base curves can be seen and the end curves resulting from the deformation can be seen in red.
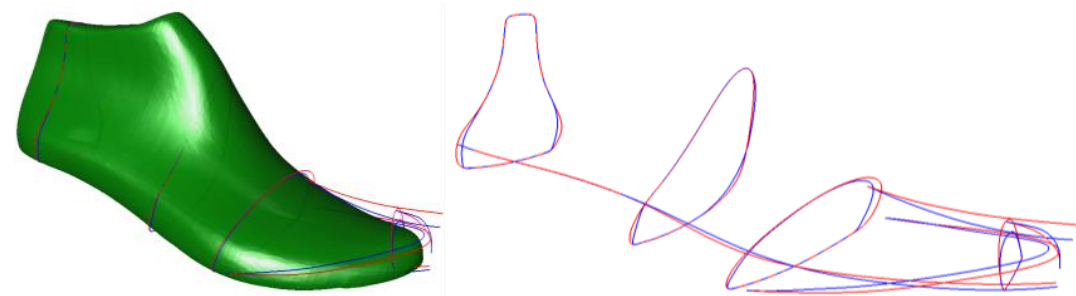

Fig. 29. Network of deformation curves and original last
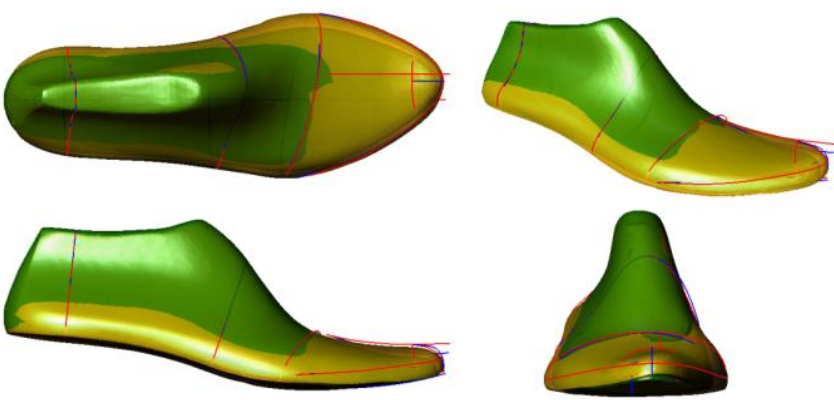

Fig. 30. Original last in green and modified last in yellow 
Step 7. Measuring and comparing the modified last.

In table 3, the measurements obtained for the reference foot, the original last and the modified last can be seen. The variation percentage of the lasts is analysed with regard to the foot along with any critical measurements that directly affect the fit.

Table 3

Comparison of foot, original last and modified last measurements (in $\mathrm{mm}$ )

\begin{tabular}{|c|c|c|c|c|c|}
\hline MEASUREMENTS & $\begin{array}{c}\text { Reference } \\
\text { Foot }\end{array}$ & $\begin{array}{c}\text { Original } \\
\text { Last }\end{array}$ & Modification & $\begin{array}{c}\text { Modified } \\
\text { Last }\end{array}$ & Gap \\
\hline SIZE & 40.0 & 40.0 & & 40.0 & \\
\hline $\begin{array}{l}\text { Distance between the feather line and the most prominent } \\
\text { point of the heel }\end{array}$ & & 2.4 & & 2.2 & \\
\hline \multicolumn{6}{|l|}{ GENERAL MEASUREMENTS } \\
\hline Last length / Foot length & 255.4 & 275.8 & & 280.5 & \\
\hline Projected width & 103.7 & 92.3 & 7.0 & 100.1 & 3.6 \\
\hline Distance to $5^{\text {th }}$ metatarsal & 156.2 & 176.1 & -16.0 & 179.0 & 2.9 \\
\hline Width of $5^{\text {th }}$ metatarsal & & 46.2 & 4.0 & 50.1 & 3.9 \\
\hline Height of CHL point (last height) & & 12.3 & 18.0 & 21.5 & 9.2 \\
\hline \multicolumn{6}{|l|}{ “HEEL" SECTION } \\
\hline Heel width & 72.5 & 63.1 & 6.0 & 69.9 & 6.8 \\
\hline \multicolumn{6}{|l|}{ "MEDIUM INSTEP" SECTION } \\
\hline Medium instep girth & 236.2 & 237.6 & 12.0 & 241.5 & 3.9 \\
\hline Medium instep width & 87.3 & 76.8 & 11.0 & 82.3 & 5.5 \\
\hline \multicolumn{6}{|l|}{ BALL GIRTH } \\
\hline $\begin{array}{l}\text { Anatomic ball girth } \\
\text { "STANDARD BALL" SECTION }\end{array}$ & 251.0 & 223.7 & 16.0 & 234.4 & 10.7 \\
\hline Section girth & 249.8 & 226.8 & 13.0 & 239.4 & 12.6 \\
\hline Section width & & 91.0 & 8.0 & 98.2 & 7.2 \\
\hline \multicolumn{6}{|l|}{ “OBLIQUE TOE” SECTION } \\
\hline Toe girth & 200.3 & 204.9 & 5.5 & 220.7 & 15.8 \\
\hline Toe width & 83.5 & 86.9 & 3.5 & 95.2 & 8.3 \\
\hline Medial toe width & & 43.8 & 3.5 & 47.2 & 3.4 \\
\hline \multicolumn{6}{|l|}{ "PERPENDICULAR TOE" SECTION } \\
\hline Perpendicular toe girth & 183.6 & 202.5 & 5.0 & 219.0 & 16.5 \\
\hline Perpendicular toe width & 77.1 & 86.3 & 2.0 & 95.1 & 8.8 \\
\hline Perpendicular toe height & & 32.1 & 4.0 & 33.8 & 1.7 \\
\hline \multicolumn{6}{|l|}{ EFFECTIVE LENGTH } \\
\hline Section height (room for $1^{\text {st }}$ toe) & 26.2 & 15.1 & 11.0 & 21.0 & 5.9 \\
\hline
\end{tabular}

In the Modification column, the modifications made to certain key measurements can bee seen, which serve as a guide to guarantee the correct fit. In the column Gap, the difference between the desired measurement and the final measurement in a certain area can be seen. These differences are evaluated by the expert user that must validate the results using his/her experience and then determine whether the modified last will be suitable for the reference foot.

Step 8. Fitting test.

After the manufacture of the new footwear style with the modified last, a test in which the user evaluates the comfort of the shoe, with regard to the previous (initial) one, is carried out subjectively. The result of this test can be seen in Table 4 where the key areas are analysed, these areas being essential for the evaluation of the fit of a last. The modifications made to the last imply a number of dimensional changes on the original last due to the pathology presented by the patient. The considerations of footwear type, the material to be used and the sole for use have been taken into account when making the modifications. All of these considerations have decisive effects on the feeling of comfort experienced by the patient, directly affecting possible subsequent modifications that will need to be made as a consequence.

Table 4

Final fit evaluations

\begin{tabular}{lcccc}
\hline & Heel & Medium instep & Ball & Toes \\
\hline Evaluation shoe model 1 & 10 & 10 & 9 & 9 \\
Evaluation shoe model 2 & 9 & 9 & 10 & 9 \\
Evaluation shoe model 3 & 9 & 10 & 10 & 8
\end{tabular}


Fig. 31 shows the physical modified last and the footwear style made with said last, with which the fitting test was carried out.
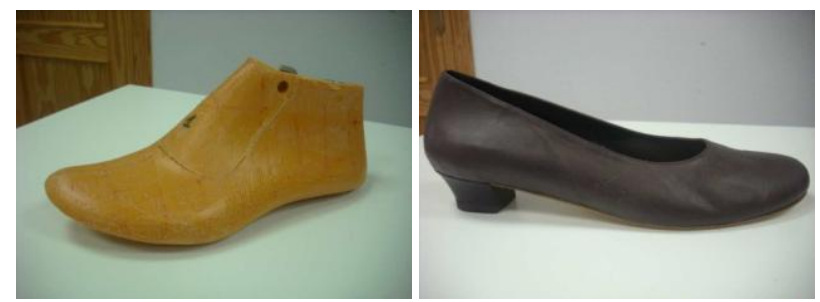

Fig. 31. Shoe model 1: Final last and shoe

In Figs. 32 and 33 two additional practical cases of shoe model application are shown. In the first one, for a men shoe, has been made a number of corrections on the original last consisting of a heel-width reduction, medium-instep adaptation and extension of the fingers area to allow better fingers movement on shoe toe zone. Comfort evaluation of this model is presented in table 4 (shoe model 2).

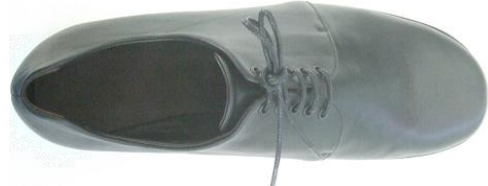

(a)

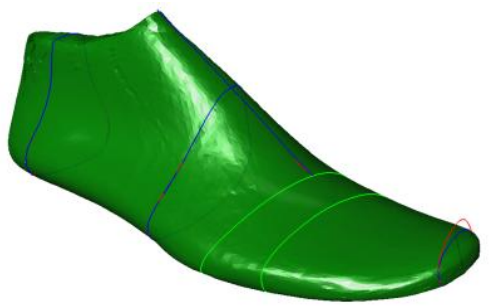

(d)

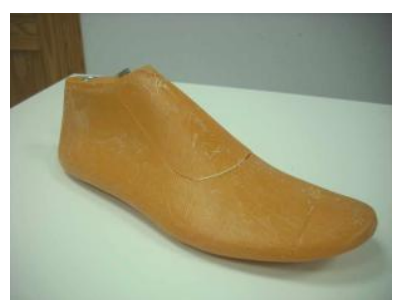

(g)

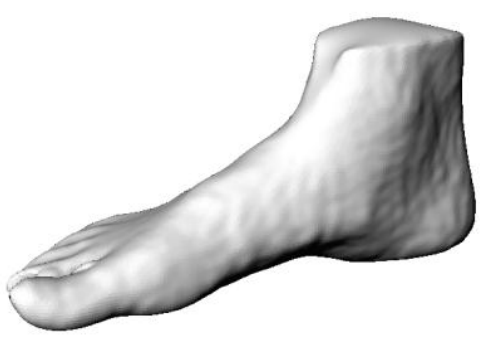

(b)

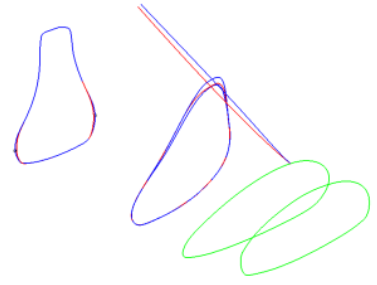

(e)

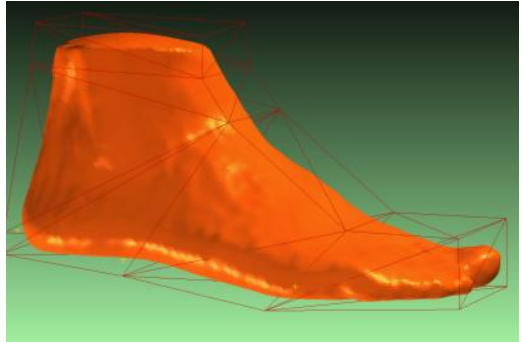

(c)
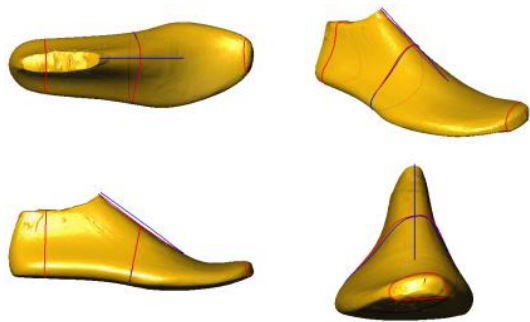

(f)

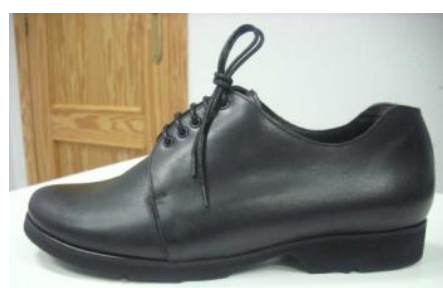

(h)

Fig. 32. Shoe model 2: Man shoe; (a) Reference shoe model, (b) Flat digitized foot, (c) Foot cage and deformation, (d) Network deformation curves and original last, (e) Network deformation curves comparative, (f) Final CAD last, (g) Final manufacturing last, (h) Final customized shoe

The next one, a women model with $75 \mathrm{~mm}$ heel height, there have been made a number of adjustments in order to fit the heel area and give a greater amplitude, expand the middle-area of the last, modify medium-instep giving greater scope, increment the area of standard ball and, finally, for aesthetic considerations has been rectified the last tip for a better design of the final customized shoe. Comfort evaluation of this model is presented in table 4 (shoe model 3). 


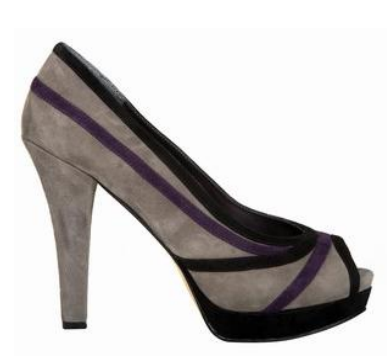

(a)

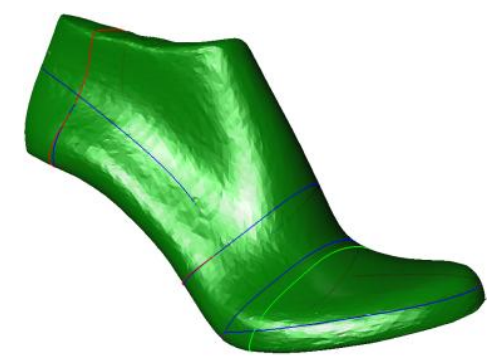

(d)

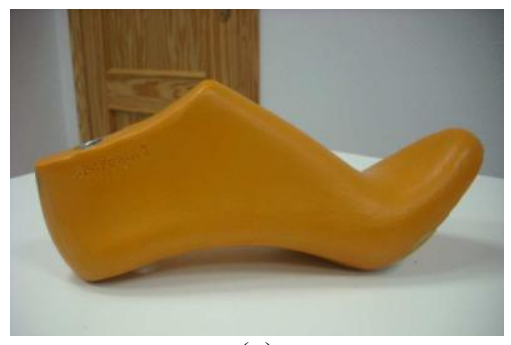

(g)

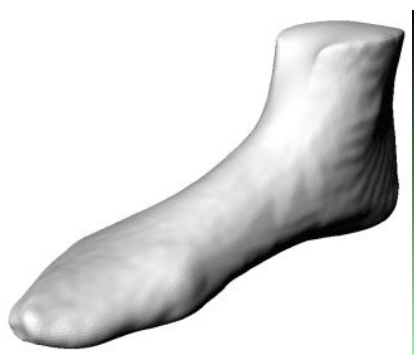

(b)

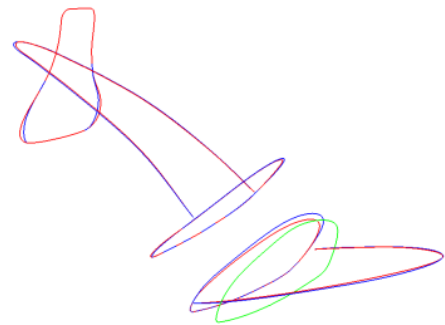

(e)

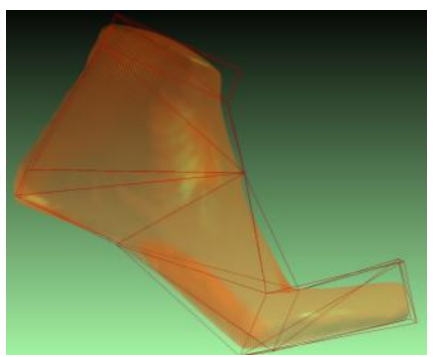

(c)

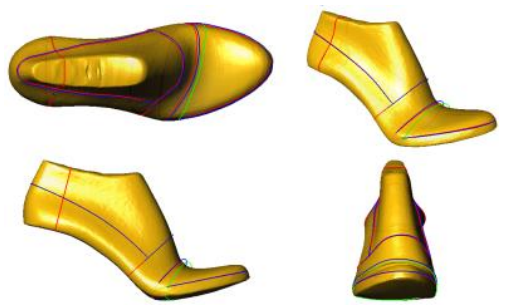

(f)

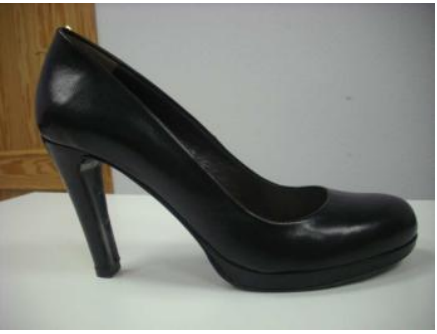

(h)

Fig. 33. Shoe model 3: Women shoe; (a) Reference shoe model, (b) Flat digitized foot, (c) Foot cage and deformation, (d) Network deformation curves and original last, (e) Network deformation curves comparative, (f) Final CAD last, (g) Final manufacturing last, (h) Final customized shoe

\section{Conclusions}

The impossibility of implementing different musculoskeletal models in the industrial field of a manufacturing sector, such as footwear, due to the complexity of real time simulation in non controlled environments, such as footwear customisation, was the starting point for this research work.

The model presented in this document interrelates two geometric models, the first allowing surface deformations to be made on a digitised foot in order to make it suitable for a last height (height derived from the heel to be incorporated into a future shoe). Foot-last adaptation is achieved through cage-based deformation techniques, using a control structure obtained through the automatic extraction of an exoskeleton from the morphometric information of the foot, which makes it possible to articulate it efficiently.

Furthermore, a last modification model has been developed that allows the adaptation of the last geometry to that of the reference foot based on quality and comfort criteria. The modification of the last was carried out through the application of a deformation technique developed in this research work and called "Network of Deformation Control Curves" $\left(N d C^{2}\right)$ which allows the precise and controlled modification of the geometry taking the quality constraints inherent to the design of this industrial element into account.

Both models have successfully been put in practice, carrying out different adaptations between the foot and last using current footwear design and manufacturing systems. In this work, a real case of footwear customisation is used to describe the adaptation of a standard last to a reference foot which presents certain conditions inherent to the user's age.

In the current system, the last modification is carried out by an expert user, with this person being the decision maker for ideal last modifications. Future works that have been proposed in this investigation will be aimed to complete the automation of the model, providing the model with an expert system which allows the adaptation of the last to the reference foot automatically and without user intervention, basing this on a series of optimisation criteria. 


\section{References}

[1] Chen, H., Nigg, B., Koning, J. (1994). Relationship between plantar pressure distribution under the foot and insole confort. Journal of Clinical Biomechanics, 9(-):335-341.

[2] Miller, J.E., Nigg, B., Liu, W., Stefanyshyn, D.J., Nurse, M.A. (2000). Influence of foot, leg and shoe characteristics on subjective comfort. International Journal of Foot and Ankle, 21(9):759-767.

[3] Jordan, C., Bartlett, R. (1995). Pressure distribution and perceived comfort in casual footwear. Journal of Gait and Posture, 3(4):215-200.

[4] Zhang, L., Helander, M.G., Drury, C.G. (1996). Identifying factors in comfort and discomfort in sitting. Journal of Human Factors, 38(3):377-389.

[5] De Looze, M., Kujit-Evers, L., Van Dieën, J. (2003). Sitting confort and disconfort and the relationships with objective measures. Journal of Ergonomics, 46(10):985-997.

[6] Au, E., Goonetilleke, R. (2007). A qualitative study on the confort and fit of ladies' dress shoes. Journal of Applied Ergonomics, 38(-):687-696.

[7] Lee, S.H., Terzopoulos, D. (2006). Heads up!: biomechanical modeling and neuromuscular control of the neck. ACM Transactions on Graphics (TOG), 25(3):1188-1198.

[8] Leng, J., Du, R. (2006). A CAD approach for designing customized shoe last. Journal of Computer Aided-Design and Applications, 3(1-4):377-384.

[9] Azariadis, P., Moulianitis, V., Alemany, S. (2007). Virtual shoe test bed: A Computer-Aided Engineering Tool for suporting shoe design. Journal of Computer-Aided Design and Applications, 4(6):741-750.

[10] Raffeli, R., Germani, M. (2011). Advanced computer aided design technologies for design automation in footwear industry. International Journal on Interactive Design and Manufacturing, 5(3):137-149.

[11] Davia, M., Germani, M., Mandolini, M., Mengoni, M., Montiel, E., Raffaeli, R. (2011). Shoes customization design tools for the diabetic foot. Computer-Aided Design and Applications, 8(5):693-711.

[12] Bernabeu, J.A., Germani, M., Mandolini, M., Megoni, M., Nester, C., Preece, S., Raffaeli, R. (2013). CAD tools for designing shoe last for people with diabetes. Original Research Article Computer-Aided Design, In Press. Online pub.

[13] Delp, S.L., Loan, J.P., Hoy, M.G., Zajac, F.E., Topp, E.L., Rosen, J.M. (1990). An interactive graphics-based model of the lower extremity to study orthopaedic surgical procedures. IEEE Transactions on bio-medical engineering, 37(8):757-767.

[14] Högfors, C., Karlsson, D., Peterson, B. (1995). Structure and internal consistency of a shoulder model. Journal of Biomechanics, 28(7):767-777

[15] Pandy, M. (2001). Computer modeling and simulation of human movement. Anual review of bio-mechanical engineering, pp. 3:245-3:273.

[16] Maural, W., Thalmann, D., Hoffmeyer, P., Beylot, P., Gingins, P., Kalra, P., Thalmann, N. (1996). A biomechanical musculoskeletal model of human upper limb for dynamic simulation. Proceedings of the Eurographics workshop on Computer animation and simulation '96. Springer-Verlag, pp. 121-136.

[17] Chao, E. (2003). Graphic-based muskuloskeletal model for biomechanical analyses and animation. Journal of Medical engineering and physics, 25(3):201-212.

[18] Blemker S.S., Delp S.L. (2005). Three-dimensional representation of complex muscle architectures and geometries. Journal of Annals of biomedical engineering, 33(5):661-673.

[19] Rasmussen, J., Damsgaard, M., Christensen, S.T., Zee, M. (2005). Anybody - decoding the human musculoskeletal system by computational mechanics. Konferanse i beregningsorientert mekanikk (computational mechanics)

[20] Wu, F., Ng-Thow-Hing, V., Singh, K., Agur, A.M., McKee, N.H. (2007). Computational representation of the aponeuroses as nurbs surfaces in $3 \mathrm{~d}$ musculoskeletal models. Computer Methods Prog. Biomedical, 88(2):112-122.

[21] Christophy, M., Faruk, N.A., Lotz, J., O’Reilly, O. (2012). A Musculoskeletal model for the lumbar spine. Biomech Model Mechanobiol, (11)19-34.
22] Scheepers, F., Parent, R.E., Carlson, W.E., May, S. F. (1997) Anatomy-based modeling of the human musculature. Proceedings of the 24th annual conference on Computer graphics and interactive techniques (SIGGRAPH '97). ACM Press/Addison-Wesley, pp. 163-172.

[23] Wilhelms, J., Van Gelder, A. (1997). Anatomically based modeling. Proceedings of the 24th annual conference on Computer graphics and interactive techniques (SIGGRAPH '97). ACM Press/Addison-Wesley, pp. 173-180.

[24] Albrecht, I., Haber, J., Seidel, H.P. (2003). Construction and animation of anatomically based human hand models. Proceedings of the 2003 ACM SIGGRAPH/Eurographics symposium on Computer animation (SCA '03). Eurographics Association, pp. 98-109.

[25] Ng-Thow-Hing, V. (2001). Anatomically-based models for physical and geometric reconstruction of humans and other animals. Ph.D. Dissertation. University of Toronto.

[26] Teran, J., Blemker, S., Hing, V., Fedkiw, R. (2003). Finite volume methods for the simulation of skeletal muscle. Proceedings of the 2003 ACM SIGGRAPH/Eurographics symposium on Computer animation (SCA '03). Eurographics Association, pp. 68-74.

[27] Zhu, Q., Chen, Y.C., Kaufman, A. (2001). Real-time biomechanically-based muscle volume deformation using FEM. Computer Graphics Forum, 17(3):275-284.

[28] Aubel, A., Thalmann, D. (2001). Interactive modeling of the human musculature. Proceedings of 14th conference of Computer Animation '01, pp. 167-255.

[29] Waters, K. (1987). A muscle model for animation threedimensional facial expression. In Proceedings of the 14th annual conference on Computer graphics and interactive techniques (SIGGRAPH '87). ACM, 21(4):17-24.

[30] Lee, Y., Terzopoulos, D., Waters, K. (1995). Realistic modelling for facial animation. Proceedings of the 22nd annual conference on Computer graphics and interactive techniques (SIGGRAPH '95). ACM, pp. 55-62.

[31] Sifakis, E., Neverov, I., Fedkiw, R. (2005). Automatic determination of facial muscle activations form sparse motion capture marker data. ACM Transactions on Graphics (TOG), 24(3):417-425

32] Shao, W., Ng-Thow-Hing, V. (2003). A general join component framework for realistic articulation in human characters. Proceedings of the 2003 symposium on Interactive $3 D$ graphics (I3D '03). ACM, pp. 11-18.

[33] Hoy, M.G., Zajac, F.E., Gordon, M.E. (1990). A musculoskeletal model of the human lower extremity: the effect of muscle, tendon, and moment arm on the momentangle relationship of musculotendon actuators at the hip, knee and ankle. Journal of Biomechanics, 23(2):157-169.

[34] Tang, Y. M., Hui, K. C. (2007). The effect of tendons on foot skin deformation. Computer Aided Design, 39(7):583-597.

[35] Tang, Y.M., Hui, K.C. (2009). Simulating tendon motion with axial mass-spring system. Journal of Computer Graphics, 33(2):162-172.

[36] Antunes, P.J., Dias, G.R., Coelho, A.T., Rebelo, F., Pereira, T. (2007). Non-linear finite element modelling of anatomically detailed $3 D$ foot model. MIMICSC.

[37] Witana, C., Goonetilleke, R., Feng J. (2004). Dimensional differences for evaluating the quality of footwear fit. Journal of Ergonomics, 47(12):1301-1317.

[38] Cheng, F.T., Perng, D.B. (1999). A systematic approach for developing a foot size information system for shoe last design. International journal of Industrial Ergonomics, 25(-):171-185.

39] Mochimaru, M., Kouchi, M. (2000). Last customization from an individual foot form and design dimensions. Journal of Ergonomics, 43(9):1301-1313.

[40] Kos, L., Duhovnik, J. (2002). A system for footwear-fitting analysis. Proceedings of International Design Conference, pp. 1187-1192.

[41] Goonetilleke, R., Luximon, A., Tsui, K. (2003a). Foot landmarking for footwear customization. Journal of Ergonomics, 46(4):364-383.

[42] Leng, J., Du, R. (2005). A deformation method for shoe last customization. Computer Aided Design and Applications, 2(14):11-18 
[43] Takahashi, Y., Hayashimoto, N., Kanamori, Y., Mitani, J., Fukui, Y., Nishihara, S. (2010). Generating a shoe last shape using laplacian deformation. Proceedings of the 8 th International Conference on Virtual Reality Continuum and its Applications in Industry (VRCAI'09). ACM, pp. 273-274.

[44] Sorkine, O., Cohen-Or, D., Lipman, Y., Alexa, M. (2004). Laplacian surface editing. Proceedings of the Eurographics/ACM SIGGRAPH Symposium on Geometry Processing. Eurographics Association, pp. 179-188.

[45] Wang, C.S. (2010). An analysis and evaluation of fitness for shoe last and human feet. Journal of Computers in Industry. Elsevier Science Publishers, 61(6):532-540.

[46] Denoth, J. (1986). Load on the locomotor system and modelling. Biomechanics of running shoes. Human Kinetic Publishers. Champign. Illinois, pp. 63-116.

[47] Perkins, P.J., Wilson, M.P. (1983). Slip resistance testing of shoes - new developments. Journal of Ergonomics, 26(1):7382 .

[48] Audemars, P. (1978). Underfoot cushioning in working footwear. A question of confort. Journal of Protection, 15(9):11-14.

[49] Hawes, M.R., Sovak, D. (1994). Quantitative morphology of human foot in a North American population. Journal of Ergonomics, 37(7):1213-1226.

[50] Ju, T., Schaefer, S., Warren, J. (2005). Mean value coodinates for closed triangular meshes. ACM Transactions on Graphics (TOG), 24(3):561-566.

[51] Floater, M. (2003). Mean value coordinates. Computer Aided Geometric Design, 20(1):19-27.

[52] Floater, M., Kos, G., Reimers, M. (2005). Mean value coordinates in 3d. Computer Aided Geometric Design, 22(7):623-631.

[53] Ju, T., Zhou, Q., Van de Panne, M., Cohen-Or, D., Neumann, U. (2008). Reusable skinning templates using cage-based deformations. ACM Transactions on Graphics (TOG), 27(5):122:1-122:10.

[54] Pratscher, M., Coleman, P., Laszlo, J., Singh, K. (2005). Outside-in anatomy based character rigging. Proceedings of the 2005 ACM SIGGRAPH/Eurographics symposium on Computer animation (SCA '05). ACM, pp. 329-338.

[55] Yoshizawa, S., Belyaev, A., Seidel, H.P. (2007). Skeletonbased variational mesh deformations. Proceedings of Computer Graphics Forum 07, pp. 255-274.

[56] Capell, S., Green, S., Curless, B., Duchamp, T., Popovic, Z. (2002). A multiresolution framework for dynamic deformations. Proceedings of the 2002 ACM SIGGRAPH/Eurographics symposium on Computer animation (SCA '02). ACM, pp. 41-47.

[57] Hormann, K., Greiner, G. (2000). MIPS: An Efficient Global Parametrization Method. Curve and surface design: SaintMalo. Vanderbilt University Press. Nashville, TN, pp. 153-162.

[58] Desbrun, M., Meyer, M., Alliez, P. (2002). Intrinsic parametrizations of surface meshes. Proceedings of Eurographics 2002 Conference, 21(3):.

[59] Khodakovsky, A.,Litke, N., Schröder, P. (2003). Globally smooth parametrizations with low distorsion. ACM Transactions on Graphics (TOG), 22(3):350-357.

[60] Schreiner, J., Asirvathan, A., Praun, E., Hoppe, H. (2004). Inter-surface mapping. Proceeding of SIGGRAPH 2004, pp. 870-877.

[61] Floater, M., Hormann, K. (2005). Surface Parameterization: a Tutorial and Survey. Advances in Multiresolution for geometric modelling, Springer-Verlag. Heidelberg, pp. 157186.

[62] Sederberg, T.W., Parry, S.R. (1986). Free-form deformation of solid geometric models. Proceedings of the 13th annual conference on Computer graphics and interactive techniques (SIGGRAPH '86), David C. Evans and Russell J. Athay (Eds.). ACM, 20(4):151-160.

[63] Coquillart, S. (1990). Extended free-form deformation. A sculpturing tool for 3D geometric modeling. SIGGRAPH Computational Graphics, 24(4):187-196.

[64] MacCracken, R., Joy, K.I. (1996). Free-form deformation with lattices of arbitrary topology. Proceedings of the 23rd annual conference on Computer graphics and interactive techniques (SIGGRAPH' '96). ACM, pp. 181-188.
[65] Kobayashi, K.G., Ootsubo, K. (2003). t-FFD free-form deformation by using triangular mesh. Proceedings of the eighth ACM symposium on Solid modeling and applications (SM '03). ACM, pp. 226-234.

[66] Floater, M. (1997). Parametrization and smooth approximation of surface triangulations. Journal of Computer Aided Geometric Design, 14(3):231-250.

[67] Floater, M. (1998). Local and global convexity preservation. Proceedings of the international conference on Mathematical methods for curves and surfaces II, pp. 183-190.

[68] Malsch, E., Dasgupta, G. (2003). Algebraic construction of smooth interpolants on polygonal domains. Proceedings of the 5th International Mathematica Symposium, 9(3):641-662.

[69] Hormann, K., Floater, M. (2006). Mean value coordinates for arbitrary planar polygons. ACM Transactions on Graphics (TOG), 25(4):1424-1441.

[70] Joshi, P., Meyer, M., DeRose, T. (2007). Harmonic coordinates for character articulation. ACM Transactions on Graphics (TOG), 26(3):71.

[71] Lipman, Y., Levin, D., Cohen-Or, D. (2008). Green coordinates. ACM Transactions on Graphics (TOG), 27(3):78:1-78:10

[72] Stoer, J., Bulirsh, R. (1992). Introduction to numerical analysis (2nd edition). Springer-Verlag. New York.

[73] Jimeno, A., Davia, M. (2011). Landmark registration for reverse design: A case study on footwear rapid prototyping. Journal of Engineering Manufacture, 225(2):235-246.

[74] Igarashi, T., Matsuoka, S., Tanaka, H. (1997). Teddy: A sketching interface for $3 \mathrm{~d}$ freeform design. ACM SIGGRAPH '07 Courses (SIGGRAPH '07). ACM, pp. Article 21.

[75] Chen, B.Y., Ono, Y., Nishita, T. (2005). Character animation creation using hand-drawn sketches. Proceedings of Pacific Graphics 2005 Conference. Journal of The Visual Computer, 21(8-10):551-558.

[76] Ji, Z., Liu, L., Chen, Z., Wang, G. (2006). Easy mesh cutting. Proceedings of Eurographics Computer Graphics Forum (CGF '06), 25(3):283-291.

[77] Kara, L.B., Shimada, K. (2006). Construction and modification of $3 \mathrm{~d}$ geometry using a sketch-based interface. Proceedings of Eurographics Workshop on Sketch-Based Interfaces and Modleing (SBM '06), pp. 45-56.

[78] Wang, H., Markosian, L. (2007). Free-form sketch. Proceedings of the 4th Eurographics workshop on Sketchbased interfaces and modeling (SBIM '07). ACM, pp. 53-58.

[79] Kho, Y., Garland, M. (2005). Sketching mesh deformations. Proceedings of the 2005 symposium on Interactive $3 D$ graphics and games (I3D '05). ACM, pp. 147-154.

[80] Nealen, A., Sorkine, O., Alexa, M., Cohen-Or, D. (2005). A sketch-based interface for detail-preserving mesh editing. ACM Transactions on Graphics (TOG), 24(3):1142-1147.

[81] Zimmermann, J., Nealen, A., Alexa, M. (2007). SilSketch: Automated sketch-based editing of surface meshes. Proceedings of the 4th Eurographics workshop on Sketchbased interfaces and modeling (SBIM '07). ACM, pp. 23-30.

[82] Yuan, X., Xu, H., Nguyen, M., Shesh, A., Chen, B. (2005). Sketch-based segmentation of scanned outdoor environment models. Proceedings of Eurographics Workshop on SketchBased Interfaces and Modleing (SBM'05), pp. 19-26.

[83] Davis, J., Agrawala, M., Chuang, E., Popovic, Z., Salesin, D. (2003). A sketching interface for articulated figure animation. Proceedings of the 2003 ACM SIGGRAPH/Eurographics symposium on Computer animation (SCA '03). Eurographics Association, pp. 320-328.

[84] Nealen, A.V. (2007). Interfaces and algorithms for the creation, modification, and optimization of surface meshes. $\mathrm{Ph} . \mathrm{D}$. Dissertation. University of Berlin.

[85] Severn, A., Faramarz, S., Costa, M. (2006). Transformation strokes. Proceedings of Eurographics Workshop on SketchBased Interfaces and Modleing (SBM '06), pp. 75-82.

[86] Singh, K., Fiume, E. (1999). Wires: A geometric deformation technique. Proceedings of the 25th annual conference on Computer graphics and interactive techniques(SIGGRAPH '98). ACM, pp. 405-414. 


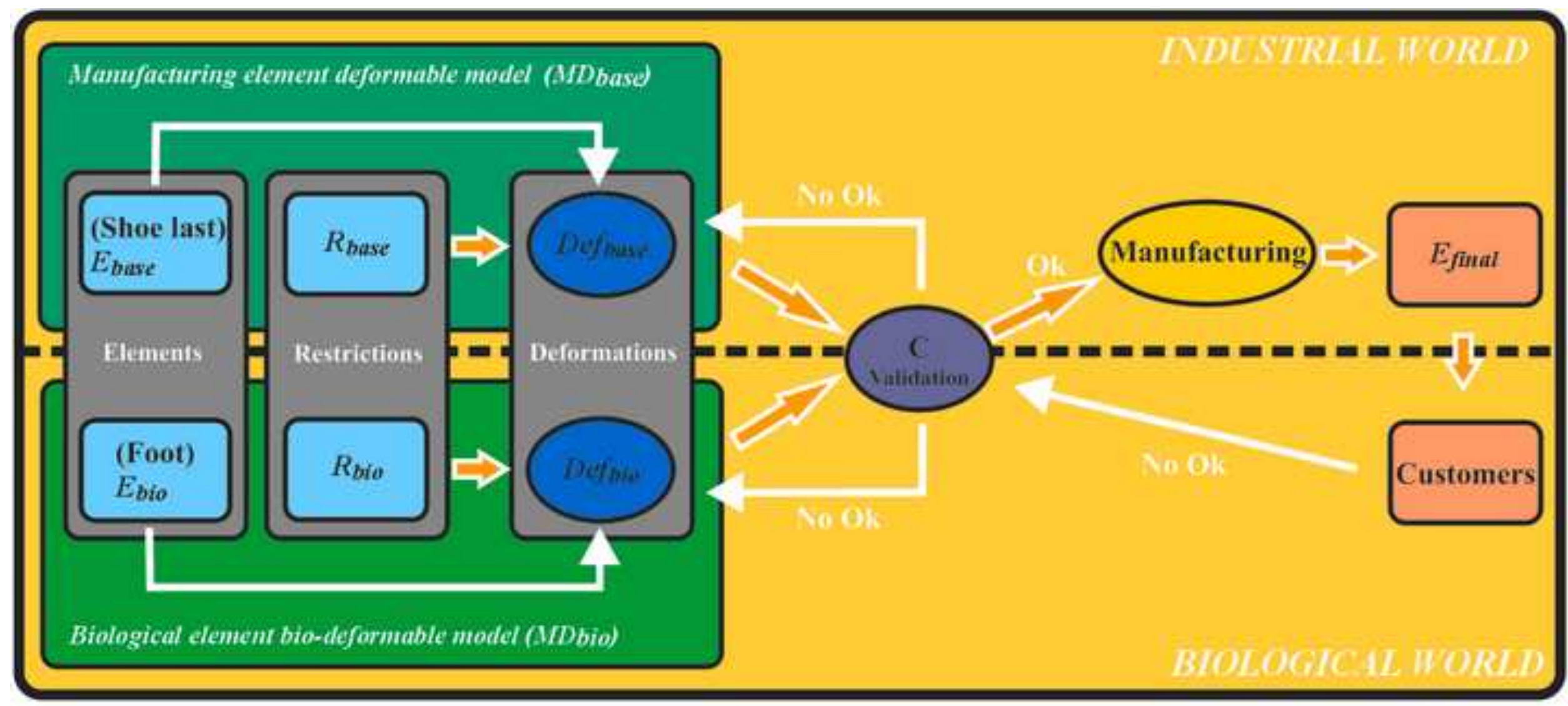




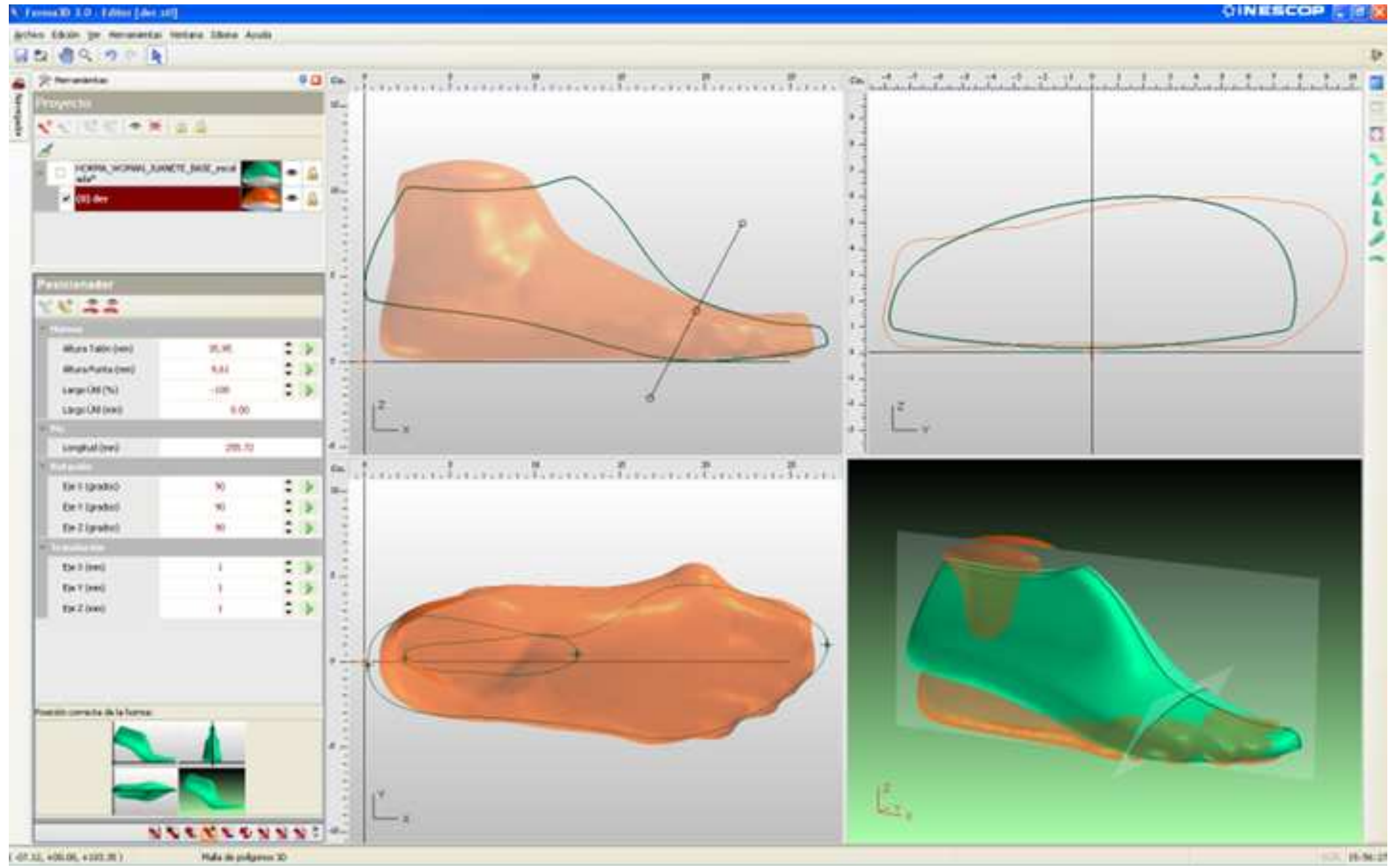


Click here to download high resolution image

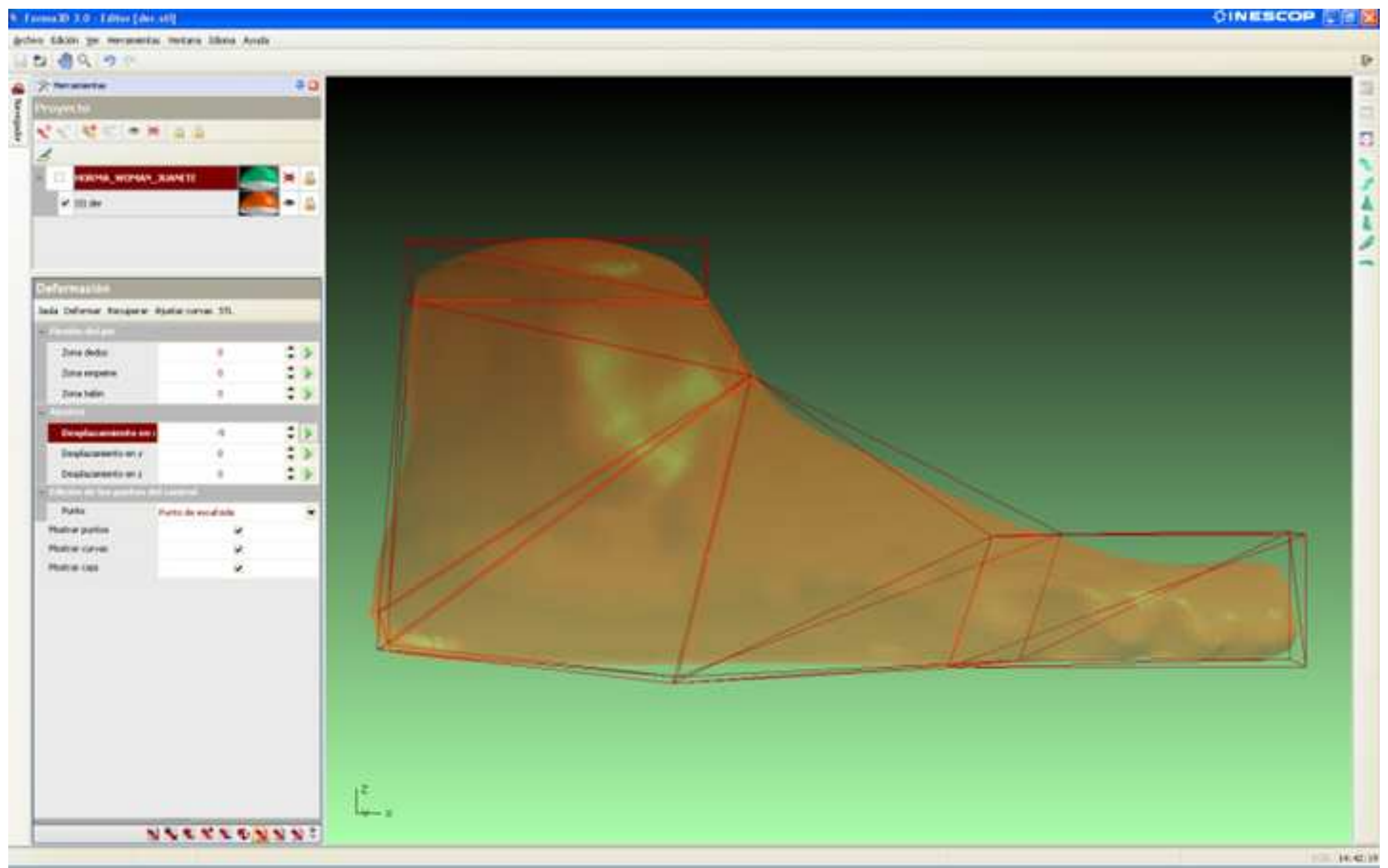




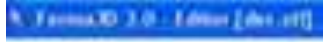

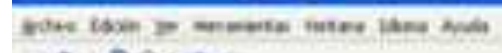

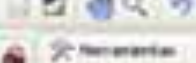

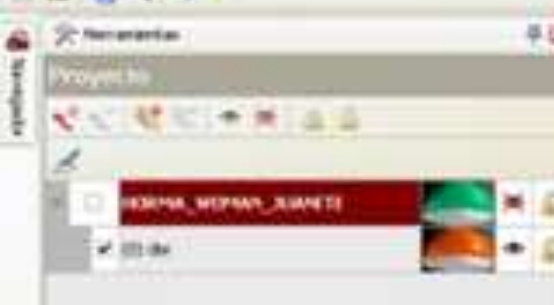

\section{Cothentis}

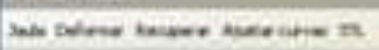

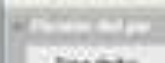

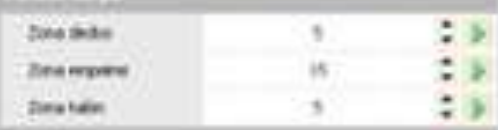

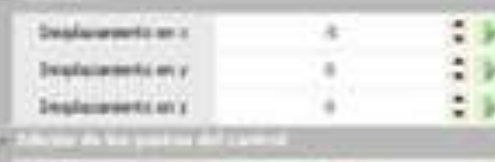

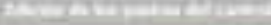

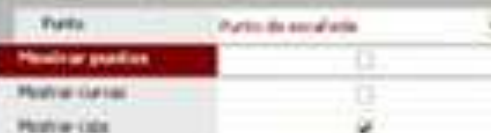

ninovias

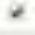

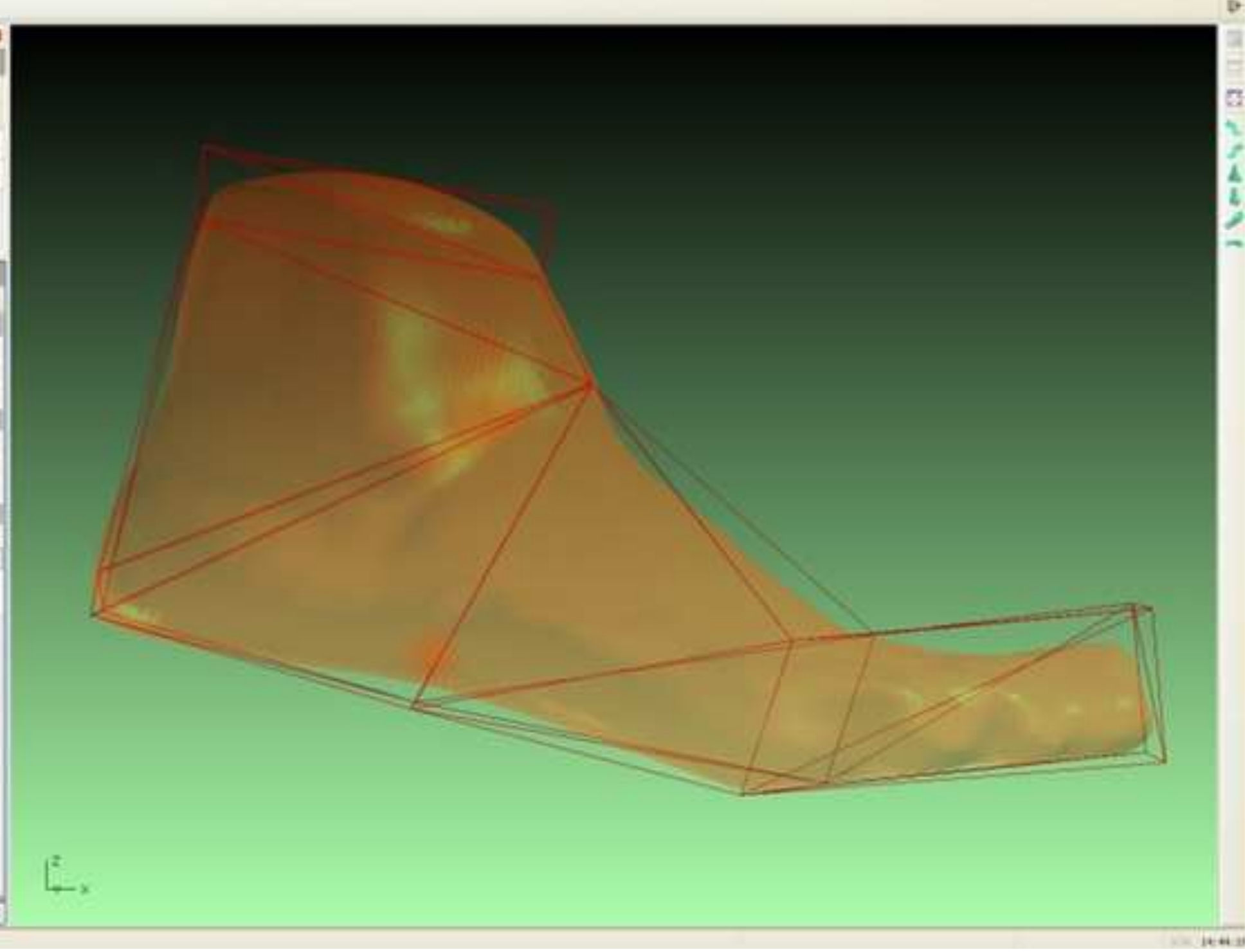


Click here to download high resolution image

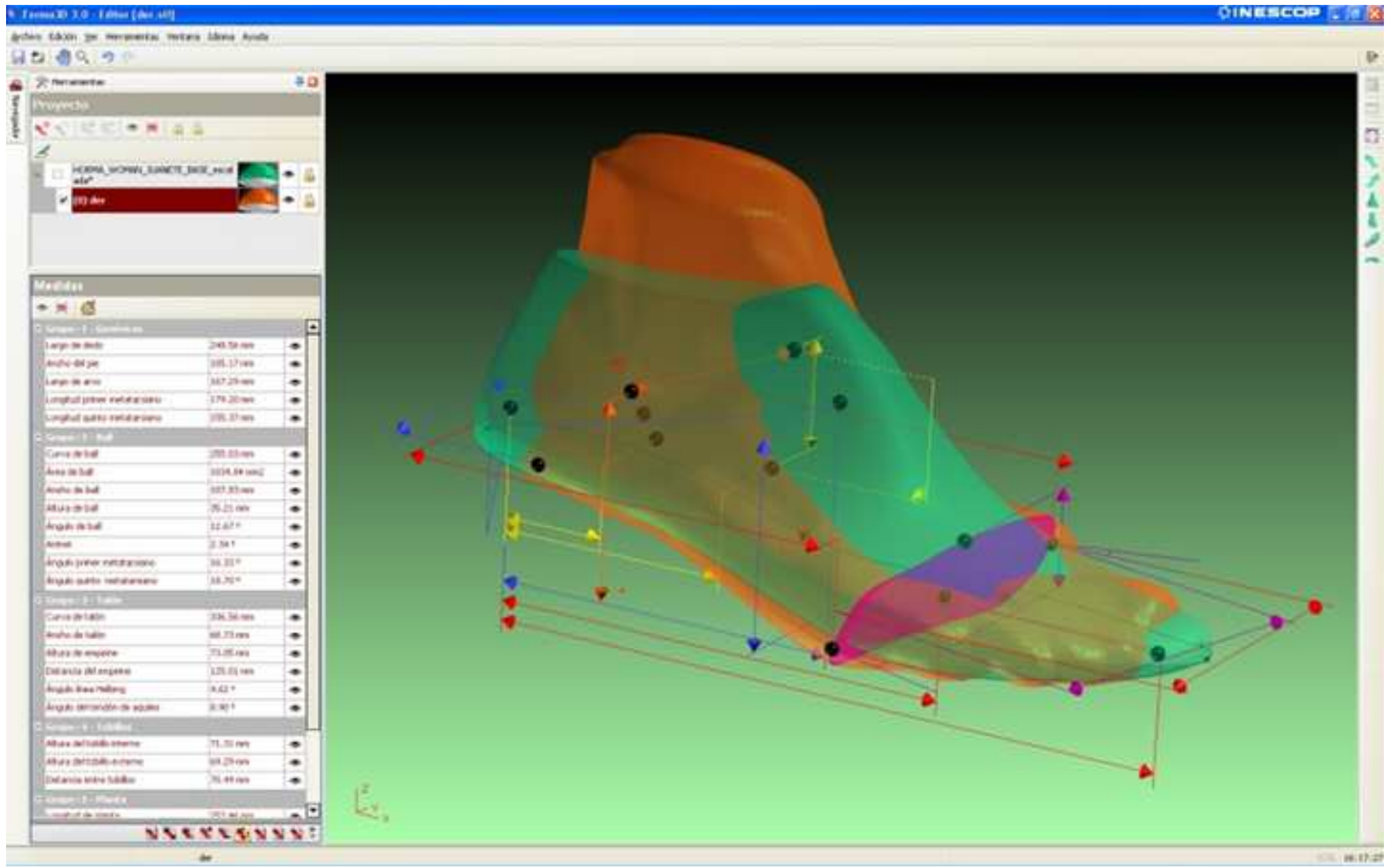


Click here to download high resolution image

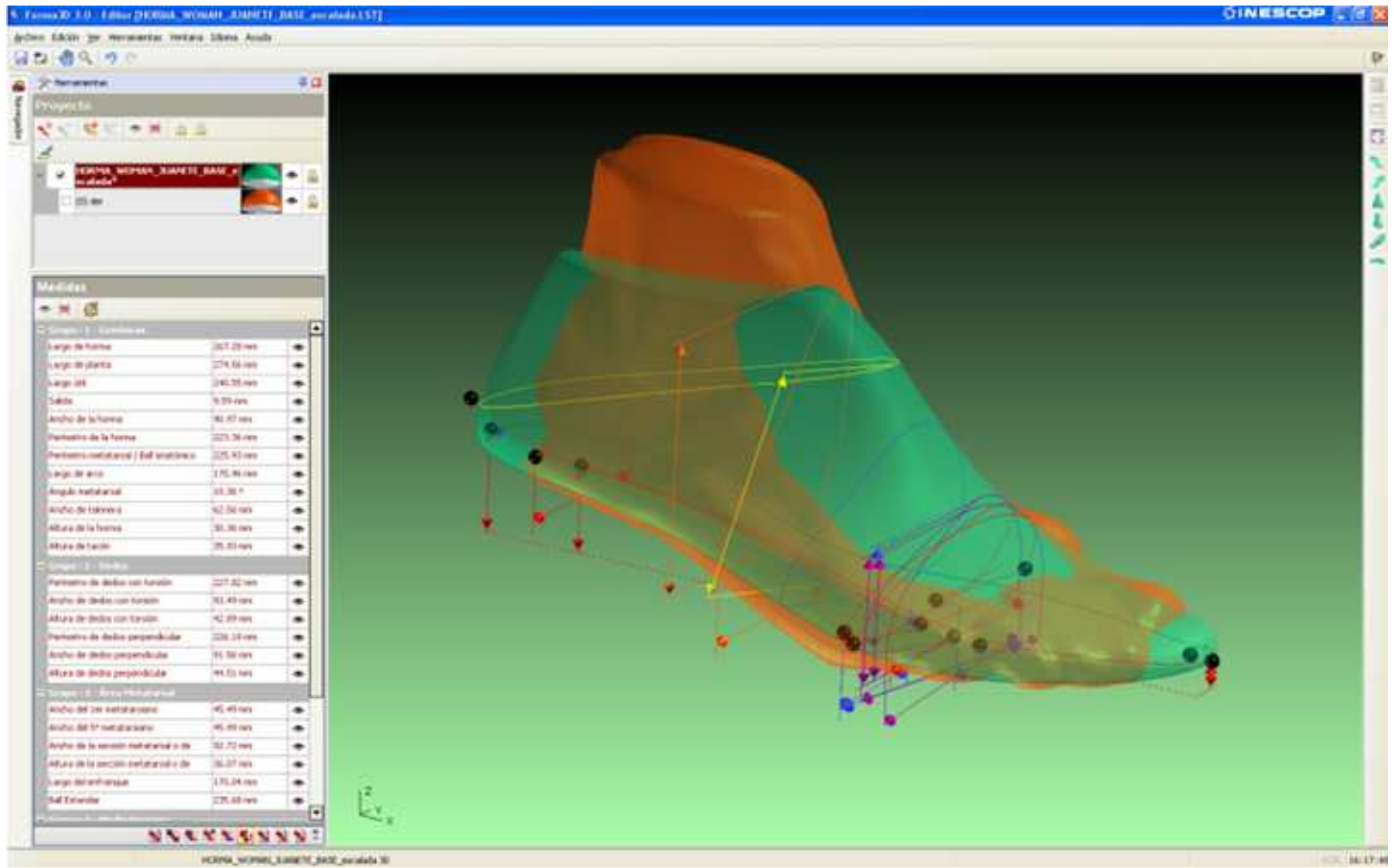

